WP ECON 18.02

\title{
Sharing the revenues from broadcasting sport events
}

\author{
Gustavo Bergantiños \\ ECOSOT, Universidade de Vigo \\ Juan D. Moreno-Ternero \\ Universidad Pablo de Olavide
}

Keywords: resource allocation, broadcasting, sport events, concede-and-divide, Shapley value

J EL Classification: D63, C71, Z20. 


\title{
Sharing the revenues from broadcasting sport events*
}

\author{
Gustavo Bergantiños ${ }^{\dagger}$ \\ Juan D. Moreno-Ternero
}

January 20, 2018

\begin{abstract}
We study the problem of sharing the revenue from broadcasting sport events, among participating players. We provide direct, axiomatic and game-theoretical foundations for two focal rules: the equal-split rule and concede-and-divide. The former allocates the revenues generated from broadcasting each game equally among the participating players in the game. The latter concedes players in each game the revenues generated by their respective fans and divides equally the residual. We also provide an application studying the case of sharing the revenue from broadcasting games in La Liga, the Spanish Football League. We show that hybrid schemes, combining our rules with lower bounds and performance measures, yield close outcomes to the current allocation being implemented by the Spanish National Professional Football League Association.
\end{abstract}

JEL numbers: D63, C71, Z20.

Keywords: resource allocation, broadcasting, sport events, concede-and-divide, Shapley value.

*Acknowledgment will be added later.

${ }^{\dagger}$ ECOSOT, Universidade de Vigo.

${ }^{\ddagger}$ Department of Economics, Universidad Pablo de Olavide 


\section{Introduction}

For most sports organizations, the sale of broadcasting and media rights is now the biggest source of revenue. A study of how much money various professional sports leagues generates shows that the NFL made $\$ 13$ billion in revenue last season. ${ }^{1}$ The Major League Baseball, came second with $\$ 9.5$ billion and the Premier League third with $\$ 5.3$ billion. ${ }^{2}$ Sharing these sizable revenues among participating teams is, by no means, a straightforward problem. Rules vary across the world. For instance, FC Barcelona and Real Madrid CF, the two Spanish giant football clubs, used to earn each more than $20 \%$ of the revenues generated by the Spanish football league. In England, however, the top two teams combined only make $13 \%$ of the revenues generated by the Premier League. ${ }^{3}$

The aim of this paper is to provide a formal model to study the problem of sharing the revenues from broadcasting sport events. Our model could be applied to different forms of competitions, but our running example will be the format of most European football leagues. That is, a round robin tournament in which each competitor (team) plays in turn against every other (home and away). Thus, the input of our model will be a (square) matrix in which each entry will be indicating the revenues associated to broadcasting the game between the two corresponding competitors. For ease of exposition, we shall assume an equal pay per view fee to each game. Thus, broadcasting revenues can be normalized to audiences.

We shall take several approaches to analyze this problem. Two rules will be salient from our analysis. On the one hand, what we shall call the equal-split rule, which allocates the revenues from each game equally among the two playing teams, and aggregates across games. On the other hand, what we shall call concede-and-divide, which concedes each team the audience from its fan base and divides equally the residual. As we shall elaborate later, both rules convey somewhat polar forms of estimating the fan effect.

More precisely, we first take a direct approach and suppose that the audience of each game

\footnotetext{
${ }^{1}$ The study "Which Professional Sports Leagues Make the Most Money" is published by Howmuch.net, a cost information website. It can be accessed at https://howmuch.net/articles/sports-leagues-by-revenue.

${ }^{2}$ Four of the top five leagues in revenue are in North America. However, 14 of the 20 biggest earners are football leagues that are mostly based in Europe.

${ }^{3}$ This might partly explain why in the last 13 editions of the Spanish Football League only once the champion was different from FC Barcelona and Real Madrid CF, whereas the Premier League witnessed 4 different champions in its last 5 editions.
} 

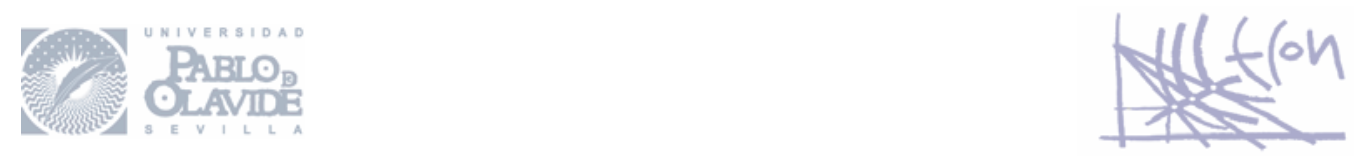

involving two teams is divided in four (disjoint) groups; namely, the generic fans of the sport being played (who watch the game, independently of the involved teams), the fans of each team (who watch the game, independently of the opponent), and the joint fans of both teams (who watch the game, because those actual two teams play). We then consider two focal scenarios for what we call the fan effect. The minimalist scenario assumes that no team has independent fans and that, therefore, the audience of each game should be equally attributed to both teams. This gives rise to the equal-split rule. The maximalist scenario assumes that teams have as many fans as possible (minimizing the number of joint fans) and, therefore, each team should be attributed the audience associated to its fan base. If generic fans of the sport being played also exist, they should be split equally, among all teams. In other words, each team concedes the other the audience attributed to its fan base, and the remainder audience is divided equally. This gives rise to concede-and-divide.

In the minimalist scenario for the fan effect described above, we also take a game-theoretical approach in which we deal with a natural cooperative game associated to the problem. It turns out that the Shapley value of such a game will always yield the same solutions as the equal-split rule for the original problem. Due to the properties of the game, the Shapley value also coincides with two other well-known values (the Nucleolus and the $\tau$-value), and it is guaranteed to be a selection of the core, which implies that the equal-split rule satisfies the standard participation constraints. In other words, the allocations provided by the equal-split rule are secession-proof, as teams do not have incentives to secede from the initial organization and create their own (sub)tournament.

In this (minimalist) scenario we also take another indirect approach in which we focus on an associated problem of adjudicating conflicting claims to the original problem. Here we observe that two of the best-known rules to adjudicate conflicting claims (the proportional and Talmud rules), which can be traced back to Aristotle and the Talmud (e.g., Moreno-Ternero and Thomson, 2017) coincide with our equal-split rule in their recommendations. The other two most well-known rules to adjudicate conflicting claims (the constrained equal-awards and constrained equal-losses rules) do not always guarantee secession-proof allocations.

As for the maximalist scenario for the fan effect, we derive concede-and-divide as an intuitive procedure of sharing audiences, partly based on a linear regression model. More precisely, if we assume the audience of a game is disentangled in four numbers, referring to the four 

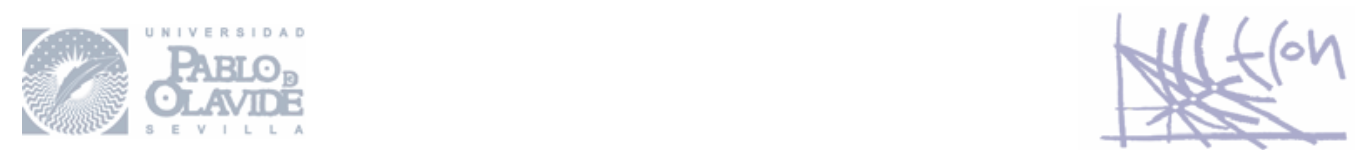

groups mentioned above, and aim to minimize the fourth (referring to the joint fans of both teams), then the problem would be equivalent to deriving the OLS estimator of a suitable linear regression model (after dealing with a problem of colinearity). We show that if we compute the OLS estimations for each of the four numbers in which an audience disentangles, the rule constructed imposing the concession of each fan base to each corresponding team, and the equal division of the remaining audience, happens to coincide with concede-and-divide.

Finally, we take an axiomatic approach to the problem formalizing axioms that reflect ethical or operational principles with normative appeal. It turns out that the two rules mentioned above are characterized by three properties. Two properties are common in both characterizations. Namely, equal treatment of equals, which states that if two competitors have the same audiences, then they should receive the same amount, and additivity, which states that revenues should be additive on the audience matrix. ${ }^{4}$ The third property in each characterization result comes from a pair of somewhat polar properties modeling the effect of null or nullifying players, respectively. More precisely, the null player property says that if nobody watches a single game of a given team (i.e., the team has a null audience), then such a team gets no revenue. On the other hand, the nullifying player property says that if a team nullifies the audience of all the games it plays with respect to a previous tournament involving the same teams (for instance, due to some kind of boycott), then the allocation of such a team should decrease exactly by the total audience of such a team in the previous tournament. ${ }^{5}$

We conclude our analysis with an empirical application focussing on La Liga, the Spanish Football League, a tournament fitting our model. We provide the schemes that our two rules would yield for the available data from that tournament. They provide reasonable (lower and upper) bounds for the amounts teams should obtain. We find that hybrid schemes, in which our rules are only used to share one fourth of the budget, whereas another fourth is allocated according to performance, and the rest half is equally split, yield close outcomes to the current allocation being implemented by the Spanish National Professional Football League.

\footnotetext{
${ }^{4} \mathrm{An}$ interpretation is that the aggregation of the revenue sharing in two seasons (involving the same competitors) is equivalent to the revenue sharing in the hypothetical combined season aggregating the audiences of the corresponding games (involving the same teams) in both seasons.

${ }^{5}$ It turns out that, as we shall show later, additivity is implied by the nullifying player property, when combined with equal treatment of equals, and, thus, it is not required in the characterization of concede-anddivide.
} 
Our work is related to several research fields, as described next.

First, it is obviously connected to the literature on sport economics. In his pioneering work within that literature, Rottenberg (1956) argued that, under the profit-maximizing assumption, revenue sharing among (sport) clubs does not affect the distribution of playing talent. ${ }^{6}$ This was later contested in more general models (e.g., Atkinson et al., 1988; Késenne, 2000). In any case, the distribution of playing talent determines the competitive balance of a sports competition and, therefore, its value (e.g., Hansen and Tvede, 2016). We are not concerned in this paper with the process of transforming revenues into playing talent that each team undertakes. Our aim, instead, is to explore appealing rules (from a normative, as well as from an empirical and game-theoretical viewpoint) to share the revenues that are collectively obtained upon selling broadcasting rights. To the best of our knowledge, this has not been addressed in the literature on sport economics yet.

Second, our work also relates to the industrial organization literature dealing with bundling. It has long been known that bundling products may increase revenue with respect to selling products independently (e.g., Adams and Yellen, 1976). Industries traditionally engaged in the practice include telecommunications, financial services, health care, and information. Transportation cards combining access to all the transportation means (e.g., bus, subway, tram) in a given city, or cultural cards doing the same for cultural venues (e.g., museums, attractions) are also frequent cases (e.g., Ginsburgh and Zang, 2003; Bergantiños and Moreno-Ternero, 2015). In our hyper-connected world, within the era of internet, new bundling strategies are emerging. Focal instances are unlimited streaming video or music downloads through periodic charges from digital video merchants or music sellers (e.g., iTunes, Netflix). There exist complex relationships between the independent price (pay per view/listening) of each product and the bundled price. Consequently, the problem of sharing the revenue from periodic charges to unlimited streaming among the participating agents (authors, artists, etc.) is a complex one. Nevertheless, it shares many features with the problem we analyze in this paper. Thus, we believe our results could shed light on that problem too.

Third, our work is connected to the axiomatic literature on resource allocation. In the last forty years, a variety of formal criteria of fair allocation have been introduced in economic theory (e.g., Thomson, 2014). These criteria have broad conceptual appeal, as well as significant

\footnotetext{
${ }^{6}$ See also El Hodiri and Quirk (1971).
} 

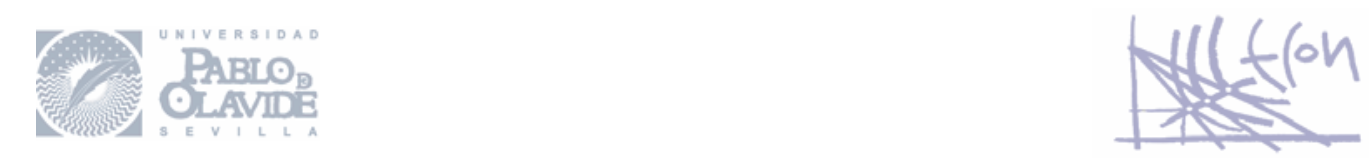

operational power, and have contributed considerably to our understanding of normative issues concerning the allocation of goods and services. The pioneering criterion was no-envy (e.g., Foley, 1967), which simply says that no agent should prefer someone else's assignment to his own. Other criteria formalizing ethical principles such as impartiality, priority, or solidarity have also played an important role in deriving fair allocation rules (e.g., Moreno-Ternero and Roemer, 2006, 2012).

Fourth, our paper is related to the literature on cooperative game theory. There is a tradition of analyzing problems involving agents' cooperation with a game-theoretical approach. Classical instances are the so-called airport problems (e.g., Littlechild and Owen, 1973), in which the cost of a runway has to be shared among different types of airplanes, bankruptcy problems from the Talmud (Aumann and Maschler, 1985), or telecommunications problems such as the Terrestrial Flight Telephone System (in short, TFTS) and the rerouting of international telephone calls (e.g., van den Nouweland et al., 1996). One of the approaches we take in this paper is precisely this one. The game we associate to our problems is formally equivalent to the game associated by van den Nouweland et al., (1996) to the TFTS situations they study. This implies that several traditional values (Shapley, Nucleolus and $\tau$-value) coincide for the game, and that they are core selections, thus guaranteeing that the participation constraints are satisfied. As we shall argue later, this constitutes a strong argument in favor of the equal-split rule (which coincides with the mentioned three values in this case).

To conclude, we mention that, in a certain sense, we can interpret a game between two teams as a joint venture with which they generate (collective) revenues. As such, the fan effect could be interpreted as a measure of each team's contribution to this joint venture. Flores-Szwagrzak and Treibich (2017) have recently introduced (and characterized) an innovative productivity index, dubbed CoScore, that disentangles individual from collaborative productivity. It is a one-parameter family capturing the endogenous relationship between individual productivity and credit allocation in the presence of teamwork. For the lowest value of the parameter, credit is allocated proportionally to each team member's endogenous score. As the value increases, the allocation becomes more egalitarian (with credit being allocated equally at the highest value). Although they apply it to formally account for coauthorship in quantifying individual scientific productivity, and to the productivity of NBA players, it is potentially applicable for our setting too, as a sort of endogenous compromise between our two polar rules. 

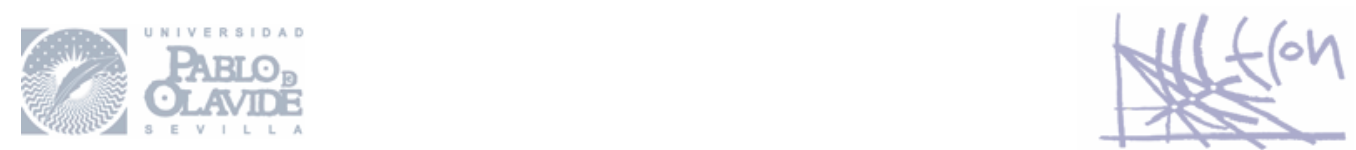

The rest of the paper is organized as follows. In Section 2, we present the model. In Section 3, we deal with the minimalist scenario for the fan effect, and two indirect approaches, which all drive towards the equal-split rule. More precisely, we take in this section a game-theoretical approach, associating a suitable cooperative game to each problem, which constitutes an endorsement for the equal-split rule. We also associate our problems to problems of adjudicating conflicting claims and appeal to focal rules in the literature dealing with these latter problems to solve the former. In Section 4, we deal with the maximalist scenario for the fan effect, which drives towards concede-and-divide. In Section 5, we present the axiomatic analysis, which supports both rules. In Section 6, we provide an empirical application to the Spanish Football League. We conclude in Section 7.

\section{The model}

Let $\mathbb{N}$ represent the set of all potential competitors (teams) and let $\mathcal{N}$ be the family of all finite (non-empty) subsets of $\mathbb{N}$. An element $N \in \mathcal{N}$ describes a finite set of teams. Its cardinality is denoted by $n$. We assume $n \geq 3 .^{7}$ Given $N \in \mathcal{N}$, let $\Pi_{N}$ denote the set of all orders on $N$. Given $\pi \in \Pi_{N}$, let $\operatorname{Pre}(i, \pi)$ denote the set of elements of $N$ which come before $i$ in the order given by $\pi$, i.e., Pre $(i, \pi)=\{j \in N \mid \pi(j)<\pi(i)\}$.

For each pair of teams $i, j \in N$, we denote by $a_{i j}$ the broadcasting audience (number of viewers) for the game played by $i$ and $j$ at $i$ 's stadium. We use the notational convention that $a_{i i}=0$, for each $i \in N$. Let $A=\left(a_{i j}\right)_{(i, j) \in N \times N}$ denote the resulting matrix with the broadcasting audiences generated in the whole tournament involving the teams within $N .{ }^{8}$ Let $\mathcal{A}_{n \times n}$ denote the set of all possible such matrices, and $\mathcal{A}=\bigcup_{n} \mathcal{A}_{n \times n}$.

For each $A \in \mathcal{A}$, let $\|A\|$ denote the total audience of the tournament. Namely,

$$
\|A\|=\sum_{i, j \in N} a_{i j}
$$

\footnotetext{
${ }^{7}$ All of our results hold under this assumption, and some of them hold too when $n=2$.

${ }^{8} \mathrm{We}$ are therefore assuming a standard round robin tournament, i.e., a league in which each team plays each other team twice: once home, another away. This is the usual format, for instance, of the main European football leagues. Our model could also be extended to encompass other formats such as those in which some teams play other teams a different number of times, or even include play-offs at the end of the regular season, which is the case of most of North American professional sports. In such a case, $a_{i j}$ would denote the broadcasting audience in all games played by $i$ and $j$ at $i$ 's stadium.
} 


\section{Dew}

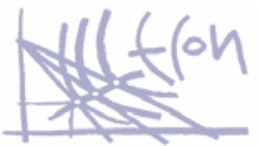

A (broadcasting sports) problem is a duplet $(N, A)$, where $N \in \mathcal{N}$ is the set of teams and $A=\left(a_{i j}\right)_{(i, j) \in N \times N} \in \mathcal{A}_{n \times n}$ is the audience matrix. The family of all the problems described as such is denoted by $\mathcal{P}$. When no confusion arises, we write $A$ instead of $(N, A)$.

For each $(N, A) \in \mathcal{P}$, and each $i \in N$, let $\alpha_{i}(N, A)$ denote the total audience achieved by team $i$, i.e.,

$$
\alpha_{i}(N, A)=\sum_{j \in N}\left(a_{i j}+a_{j i}\right)
$$

When no confusion arises, we write $\alpha_{i}$ instead of $\alpha_{i}(N, A)$. Notice that, for each problem $(N, A) \in \mathcal{P}, \sum_{i \in N} \alpha_{i}(N, A)=2\|A\|$.

Consider the following example, to which we will after refer.

Example 1 Let $(N, A) \in \mathcal{P}$ be such that $N=\{1,2,3\}$ and

$$
A=\left(\begin{array}{ccc}
0 & 1200 & 1030 \\
1200 & 0 & 230 \\
1030 & 230 & 0
\end{array}\right)
$$

Then, $\|A\|=4920$ and $\alpha(N, A)=\left(\alpha_{1}(N, A), \alpha_{2}(N, A), \alpha_{3}(N, A)\right)=(4460,2860,2520)$.

A (sharing) rule is a mapping that associates with each problem an allocation indicating the amount each team gets from the total revenue generated by broadcasting games. Without loss of generality, we normalize the revenue generated from each viewer to 1 (to be interpreted as the "pay per view" fee). Thus, formally, $R: \mathcal{P} \rightarrow \mathbb{R}^{n}$ is such that, for each $(N, A) \in \mathcal{P}$,

$$
\sum_{i \in N} R_{i}(N, A)=\|A\|
$$

An obvious example of rule is the one that simply divides the total audience equally among the teams. This rule ignores differences between teams and, thus, we shall discard it. The next two, which will be the main focus of this work, do not. They share a common ground as they take as starting point each team's total audience $\left(\alpha_{i}\right)$. They both subtract from it an amount associated to each of the remaining $n-1$ teams. For the first rule, an equal share of half of the team's total audience $\left(\beta_{i}\right)$. For the second rule, the average audience per game that the remaining teams yield in the remaining games they play $\left(\gamma_{i}\right)$. Formally,

Equal-split rule, $E S$ : for each $(N, A) \in \mathcal{P}$, and each $i \in N$,

$$
E S_{i}(N, A)=\alpha_{i}-(n-1) \beta_{i}=\frac{\alpha_{i}}{2}=\frac{\sum_{j \in N}\left(a_{i j}+a_{j i}\right)}{2} .
$$




\section{Dew}

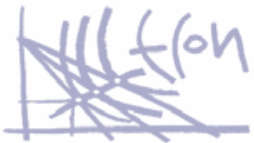

Concede-and-divide, $C D$ : for each $(N, A) \in \mathcal{P}$, and each $i \in N$,

$$
C D_{i}(N, A)=\alpha_{i}-(n-1) \gamma_{i}=\alpha_{i}-\frac{\sum_{j, k \in N \backslash\{i\}}\left(a_{j k}+a_{k j}\right)}{n-2} .
$$

The equal-split rule therefore splits equally the audience of each game. Alternatively, one could say that it divides the total audience proportionally to the vector of audiences of the teams. In that sense, the rule is endorsing one of the most widely accepted principles of distributive justice (namely, proportionality), which can be traced back to Aristotle. ${ }^{9}$

Concede-and-divide, on the other hand, compares the performance of a team with the average performance of the other teams. ${ }^{10}$ It turns out that it is equivalent to a specific linear combination of the equal-split rule and the rule that divides the total audience equally among the teams. Namely, for each $(N, A) \in \mathcal{P}$, and each $i \in N$,

$$
C D_{i}(N, A)=\frac{(n-1) \alpha_{i}-\|A\|}{n-2}=\frac{2(n-1)}{n-2} E S_{i}(N, A)-\frac{n}{n-2} \frac{\|A\|}{n} .
$$

In Example 1 we have that

\begin{tabular}{|c|c|c|c|}
\hline Rule/Team & 1 & 2 & 3 \\
\hline Equal-Split & 2230 & 1430 & 1260 \\
\hline Concede-and-Divide & 4000 & 800 & 120 \\
\hline
\end{tabular}

The equal-split rule ignores the existence of team fans. It splits the audience of each game equally among the two participating teams. Now, suppose a given game between teams $i$ and $j$ is watched (via broadcasting) by, say, 200 people. Given our normalizing assumption, this means the game generates a revenue of 200. Imagine now we have the following additional information: 20 individuals watched the game simply because they like the sport (and might have watched any game); 100 individuals watched the game because they are fans of team $i$, whereas 30 individuals watched the game because they are fans of team $j$. Finally, the remaining 50 individuals watched the game because they considered that particular game between teams $i$ and $j$ was appealing. The equal-split rule would propose awarding 100 to each team, thus ignoring the unequal number of fans of the two teams. An alternative allocation, taking into account this latter aspect, would concede each team the amount generated by its fans (100 for

\footnotetext{
${ }^{9}$ The reader is referred to Section 3.2 where we elaborate further on this point.

${ }^{10} \mathrm{~A}$ plausible reason to name this rule as such will be given later in this section.
} 

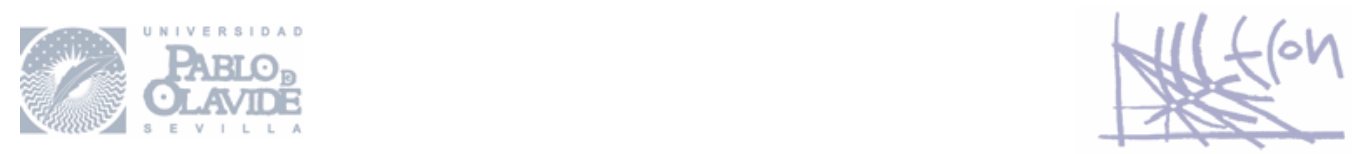

team $i, 30$ for team $j$ ) and would divide the rest equally. That is, team $i$ would receive 135 whereas team $j$ would receive 65 . As we shall see next, concede-and-divide, which can be traced back to the Talmud, paves the way for the rule we defined above under the same name. ${ }^{11}$

The fan effect described above is relevant. It might actually explain (at least, partially) why audiences differ so much. Some teams have more fans than others and, consequently, they generate larger audiences. This aspect seems to be indeed taken into account by the actual revenue sharing rules used in professional sports, where the amount assigned to each team depends on some parameters that try to capture such differences.

We can safely argue that, in general, one might become a viewer of a game involving teams $i$ and $j$ for several reasons:

1. Being a fan of this sport per se (in which case one would be eager to watch all the games, independently of the teams playing).

2. Being a fan of team $i$ (in which case one would be eager to watch all the games involving team $i)$.

3. Being a fan of team $j$ (in which case one would be eager to watch all the games involving team $j)$.

4. Considering that the game between teams $i$ and $j$ is interesting.

In practice, the above information is not available and we only know the total audience of the game. Let us, for instance, revisit Example 1. Therein, we can conjecture several plausible hypothesis (in terms of items 1 to 4 described above) for the provided audiences.

Hypothesis $(a)$. All viewers belong to group 4 and, thus, no team has fans. In this case, the procedure described above (conceding each game its fan base and dividing equally the remainder) would recommend awarding team 1

$$
\frac{1200}{2}+\frac{1030}{2}+\frac{1200}{2}+\frac{1030}{2}=2230
$$

The allocation would be

$$
(2230,1430,1260)
$$

\footnotetext{
${ }^{11}$ The term was coined by Thomson (2003) to illustrate the solution to the so-called contested-garment problem appearing in the Talmud.
} 


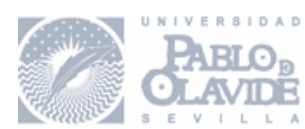

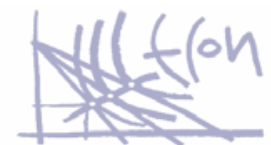

which is the allocation proposed by the equal-split rule.

$\underline{\text { Hypothesis }(b)}$. Team 1 has 1000 fans, team 2 has 200 fans and team 3 has 30 fans. No viewers belong to groups 1 or 4 . In this case, the procedure described above would recommend awarding 4000 to team 1 (it plays 4 games with 1000 fans in each). The allocation would be

$$
(4000,800,120)
$$

which is the allocation proposed by concede-and-divide.

Hypothesis (c). Team 1 has 800 fans, team 2 has 100 fans and team 3 has 30 fans. 90 viewers belong to group 1. The remaining viewers belong to group 4. That is,

\begin{tabular}{|c|c|c|c|c|c|}
\hline Totals & Group 1 & Fans 1 & Fans 2 & Fans 3 & Group 4 \\
\hline 1200 & 90 & 800 & 100 & & 210 \\
\hline 1030 & 90 & 800 & & 30 & 110 \\
\hline 230 & 90 & & 100 & 30 & 10 \\
\hline
\end{tabular}

In this case, the procedure described above would recommend the allocation

$$
(3700,800,420)
$$

which is somewhat in between the other two allocations described above.

The first two hypothesis described above can be thought of as the extreme scenarios regarding the fan effect. They can be generalized as follows:

In the first (minimalist) scenario, it is assumed that there are no fans. Thus, it seems natural to divide $a_{i j}$ equally between teams $i$ and $j$, for each $i, j \in N$. This is what the equal-split rule recommends.

In the second (maximalist) scenario, we assume there exist as many fans as possible, compatible with the data. We do so by minimizing the number of viewers in group 4. As we shall see later, this is what concede-and-divide recommends.

The two rules therefore provide polar estimates of the fan effect. Based on this, we argue that they should provide a range in which allocations estimating the fan effect should lie. For instance, in Example 1, team 1 should receive something between 2230 and 4000, team 2 between 800 and 1430 and team 3 between 120 and 1260 . 

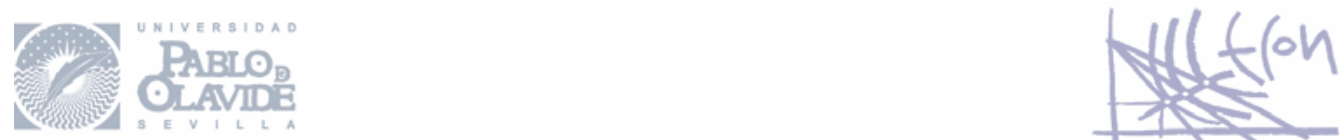

\section{The minimalist scenario for the fan effect and the equal-split rule}

In this section we consider the lower bound for the fan effect. In the parlance used above, we assume that all viewers belong to group 4 and, therefore, that teams have no fans. This means that a person decides to watch a game only because of the pair of teams playing the game.

In this scenario, and as argued above, it seems natural to consider the equal-split rule. We analyze it here in detail. In the first subsection, we associate to each problem a cooperative game with transferable utility. We prove that the Shapley value of the game coincides with the equal-split rule. The core is non empty and the equal-split rule belongs to the core, and this is noteworthy because the Shapley value is not always in the core. In the second subsection, we associate to each problem a claims problem. We prove that the so-called proportional and Talmud rules for claims problems coincide with the equal-split rule.

\subsection{The (cooperative) game-theoretical approach}

A cooperative game with transferable utility, briefly a TU game, is a pair $(N, v)$, where $N$ denotes a set of agents and $v: 2^{N} \rightarrow \mathbb{R}$ satisfies $v(\varnothing)=0$. We say that $(N, v)$ is convex if, for each pair $S, T \subset N$ and $i \in N$ such that $S \subset T$ and $i \notin T$,

$$
v(T \cup\{i\})-v(T) \geq v(S \cup\{i\})-v(S) .
$$

We present some well-known solutions for TU games. First, the core, defined as the set of feasible payoff vectors, upon which no coalition can improve. Formally,

$$
C(N, v)=\left\{x \in \mathbb{R}^{N} \text { such that } \sum_{i \in N} x_{i}=v(N) \text { and } \sum_{i \in S} x_{i} \geq v(S) \text {, for each } S \subset N\right\} \text {. }
$$

The Shapley value (Shapley, 1953) is defined for each player as the average of his contributions across orders of agents. Formally, for each $i \in N$,

$$
S h_{i}(N, v)=\frac{1}{n !} \sum_{\pi \in \Pi_{N}}[v(\operatorname{Pre}(i, \pi) \cup\{i\})-v(\operatorname{Pre}(i, \pi))]
$$

We associate with each (broadcasting sports) problem $(N, A) \in \mathcal{P}$ a TU game $\left(N, v_{A}\right)$ where, for each $S \subset N, v_{A}(S)$ denotes the total audience of the games played by the teams in 
S. Namely,

$$
v_{A}(S)=\sum_{\substack{i, j \in S \\ i \neq j}} a_{i j}=\sum_{\substack{i, j \in S \\ i<j}}\left(a_{i j}+a_{j i}\right) .
$$

Notice that, for each problem $(N, A) \in \mathcal{P}$ and each $i \in N, v_{A}(\{i\})=0$.

In Example 1,

$$
\begin{array}{ccccc}
S & \{1,2\} & \{1,3\} & \{2,3\} & \{1,2,3\} \\
v_{A}(S) & 2400 & 2060 & 460 & 4920
\end{array}
$$

and

$$
\operatorname{Sh}\left(N, v_{A}\right)=(2230,1430,1260)=E S(N, A)
$$

The next result establishes a correspondence between the equal-split rule and the Shapley value for the TU-game described above.

Theorem 1 For each $(N, A) \in \mathcal{P}, E S(N, A)=S h\left(N, v_{A}\right)$.

Proof. Let $(N, A) \in \mathcal{P}$ and $\left(N, v_{A}\right)$ be its associated TU game. For each pair $i, j \in N$ with $i \neq j$ we define the function $v_{A}^{i j}$ as follows. For each $S \subset N$,

$$
v_{A}^{i j}(S)=\left\{\begin{array}{cc}
a_{i j}+a_{j i} & \text { if }\{i, j\} \subset S \\
0 & \text { otherwise }
\end{array}\right.
$$

Consider the resulting TU-game $\left(N, v_{A}^{i j}\right)$. It is straightforward to see that, for such a game, agents $i$ and $j$ are symmetric, whereas the remaining agents in $N \backslash\{i, j\}$ are null. Thus,

$$
S h_{k}\left(N, v_{A}^{i j}\right)=\left\{\begin{array}{cc}
\frac{a_{i j}+a_{j i}}{2} & \text { if } k \in\{i, j\} \\
0 & \text { otherwise. }
\end{array}\right.
$$

For each $S \subset N$,

$$
v_{A}(S)=\sum_{\substack{i, j \in S \\ i<j}}\left(a_{i j}+a_{j i}\right)=\sum_{\substack{i, j \in N \\ i<j}} v_{A}^{i j}(S) .
$$

As the Shapley value is additive on $v$, we have that

$$
S h\left(N, v_{A}\right)=\sum_{\substack{i, j \in N \\ i<j}} S h\left(N, v_{A}^{i j}\right) .
$$


Thus, for each $k \in N$,

$$
S h_{k}\left(N, v_{A}\right)=\sum_{\substack{i, j \in N \\ i<j}} S h_{k}\left(N, v_{A}^{i j}\right)=\sum_{j \in N} S h_{k}\left(N, v_{A}^{k j}\right)=\sum_{j \in N} \frac{a_{k j}+a_{j k}}{2}=\frac{\alpha_{k}}{2} .
$$

The game we have described in this section is formally equivalent to the game associated by van den Nouweland et al., (1996) to the so-called Terrestial Flight Telephone System (in short, TFTS) situations they formalize. ${ }^{12}$ They prove that such a game is convex and, therefore, its Shapley value belongs to the core (e.g., Shapley, 1953). Thus, it follows from Theorem 1 that the equal-split rule always yields secession-proof allocations. Formally, $E S(N, A) \in C\left(N, v_{A}\right)$, for each problem $(N, A)$.

The next result fully characterizes the core of this game.

Proposition 1 Let $(N, A) \in \mathcal{P}$ and $\left(N, v_{A}\right)$ be its associated TU game. Then, $x=\left(x_{i}\right)_{i \in N} \in$ $C\left(N, v_{A}\right)$ if and only if, for each $i \in N$, there exist $\left(x_{i}^{j}\right)_{j \in N \backslash\{i\}}$ satisfying three conditions:

(i) $x_{i}^{j} \geq 0$, for each $j \in N \backslash\{i\}$;

(ii) $\sum_{j \in N \backslash\{i\}} x_{i}^{j}=x_{i}$;

(iii) $x_{i}^{j}+x_{j}^{i}=a_{i j}+a_{j i}$, for each $j \in N \backslash\{i\}$;

Proof. We first prove that if $x=\left(x_{i}\right)_{i \in N}$ is such that, for each $i \in N$, there exists $\left(x_{i}^{j}\right)_{j \in N \backslash\{i\}}$ satisfying the three conditions, then $x \in C\left(N, v_{A}\right)$.

By (ii),

$$
\sum_{i \in N} x_{i}=\sum_{i \in N} \sum_{j \in N \backslash\{i\}} x_{i}^{j}=\sum_{\substack{i, j \in N \\ i<j}}\left(x_{i}^{j}+x_{j}^{i}\right)
$$

By (iii),

$$
\sum_{\substack{i, j \in N \\ i<j}}\left(x_{i}^{j}+x_{j}^{i}\right)=\sum_{\substack{i, j \in N \\ i<j}}\left(a_{i j}+a_{j i}\right)=v_{A}(N) .
$$

Analogously, for each $S \subset N$,

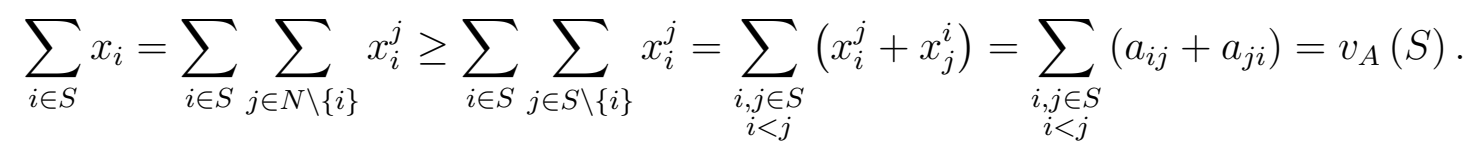

\footnotetext{
${ }^{12}$ A Terrestrial Flight Telephone System refers to an agreement made by a group of countries in order to provide a network of ground stations so that phone calls can be made within their airplanes while flying above their territory.
} 
Then, $x \in C\left(N, v_{A}\right)$.

Conversely, let $x=\left(x_{i}\right)_{i \in N} \in C\left(N, v_{A}\right)$. As $\left(N, v_{A}\right)$ is convex, the core is the convex hull of the vectors corresponding to the various actions in the player set. Thus, there exists $\left(y_{\pi}\right)_{\pi \in \Pi_{N}}$ with $y_{\pi} \geq 0$ for each $\pi \in \Pi_{N}$ and $\sum_{\pi \in \Pi_{N}} y_{\pi}=1$ such that, for each $i \in N$,

$$
x_{i}=\sum_{\pi \in \Pi_{N}} y_{\pi}\left[v_{A}(\operatorname{Pre}(i, \pi) \cup\{i\})-v_{A}(\operatorname{Pre}(i, \pi))\right] .
$$

Because of the definition of $v_{A}$, we have that

$$
x_{i}=\sum_{\pi \in \Pi_{N}} y_{\pi}\left[\sum_{j \in \operatorname{Pre}(i, \pi)}\left(a_{i j}+a_{j i}\right)\right]=\sum_{j \in N \backslash\{i\}}\left(a_{i j}+a_{j i}\right) \sum_{\pi \in \Pi_{N}, j \in \operatorname{Pre}(i, \pi)} y_{\pi} .
$$

For each pair $i, j \in N$, with $i \neq j$, we define

$$
x_{i}^{j}=\left(a_{i j}+a_{j i}\right) \sum_{\pi \in \Pi_{N}, j \in \operatorname{Pre}(i, \pi)} y_{\pi} .
$$

Thus, $x_{i}^{j} \geq 0$, for each $j \in N \backslash\{i\}$, and for each $i \in N$, i.e., $(i)$ holds.

Furthermore, $\sum_{j \in N \backslash\{i\}} x_{i}^{j}=x_{i}$, i.e., (ii) holds.

Let $i, j \in N$ with $i \neq j$. Then,

$$
\begin{aligned}
x_{i}^{j}+x_{j}^{i} & =\left(\left(a_{i j}+a_{j i}\right) \sum_{\pi \in \Pi_{N}, j \in \operatorname{Pre}(i, \pi)} y_{\pi}\right)+\left(\left(a_{i j}+a_{j i}\right) \sum_{\pi \in \Pi_{N}, i \in \operatorname{Pre}(j, \pi)} y_{\pi}\right) \\
& =\left(a_{i j}+a_{j i}\right) \sum_{\pi \in \Pi_{N}} y_{\pi}=a_{i j}+a_{j i},
\end{aligned}
$$

i.e., (iii) holds.

The above proposition states that, in order to satisfy the core constraints, we should divide the revenue generated by the audience of a game between the two teams playing the game. There is complete freedom within those bounds. For instance, assigning all the revenue to one of the teams is admissible. The equal-split rule states that the revenue generated by the audience of a game be divided equally between the two teams playing the game. Thus, the allocations that the equal-split rule yields satisfy the core constraints, as mentioned above.

This is a strong argument in favor of the equal-split rule. Teams are corporations and, as such, any subgroup of teams could potentially secede and form another (smaller) competition. Thus, if the rule selects allocations within the core, it provides stable outcomes. As shown above, in this case, the core is non-empty and very large. Thus, it seems reasonable to select one allocation within the core. 

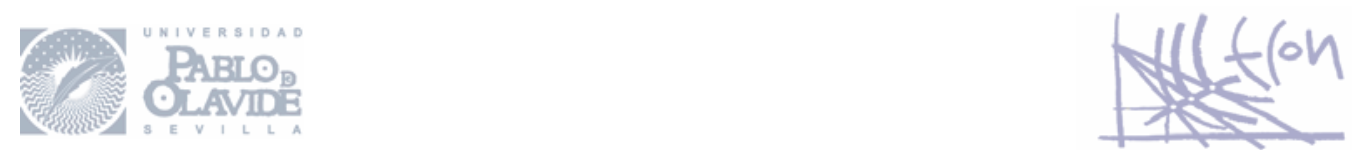

\subsection{The conflicting claims approach}

O'Neill (1982) is credited for introducing one of the simplest (and yet useful) models to study distributive justice. The so-called problem of adjudicating conflicting claims refers to a situation in which an amount of a perfectly divisible good (the endowment) has to be allocated among a group of agents who hold claims against it, and the aggregate claim is higher than the endowment. This basic framework is flexible enough to accommodate a variety of related situations that trace back to ancient sources such as Aristotle's essays and the Talmud. ${ }^{13}$ It turns out that, as we show in this section, our problems could also be seen as a specific instance of the problem of adjudicating conflicting claims.

Formally, a problem of adjudicating conflicting claims (or, simply, a claims problem) is a triple consisting of a population $N \in \mathcal{N}$, a claims profile $c \in \mathbb{R}_{+}^{n}$, and an endowment $E \in \mathbb{R}_{+}$ such that $\sum_{i \in N} c_{i} \geq E$. Let $C \equiv \sum_{i \in N} c_{i}$. To avoid unnecessary complication, we assume $C>0$. Let $\mathcal{D}^{N}$ be the domain of claims problems with population $N$ and $\mathcal{D} \equiv \bigcup_{N \in \mathcal{N}} \mathcal{D}^{N}$.

Given a problem $(N, c, E) \in \mathcal{D}^{N}$, an allocation is a vector $x \in \mathbb{R}^{n}$ satisfying the following two conditions: (i) for each $i \in N, 0 \leq x_{i} \leq c_{i}$ and (ii) $\sum_{i \in N} x_{i}=E$. We refer to (i) as boundedness and (ii) as balance. A rule on $\mathcal{D}, R: \mathcal{D} \rightarrow \bigcup_{N \in \mathcal{N}} \mathbb{R}^{n}$, associates with each problem $(N, c, E) \in \mathcal{D}$ an allocation $R(N, c, E)$ for the problem.

The constrained equal-awards rule, $C E A$, selects, for each $(N, c, E) \in \mathcal{D}$, the vector $\left(\min \left\{c_{i}, \lambda\right\}\right)_{i \in N}$, where $\lambda>0$ is chosen so that $\sum_{i \in N} \min \left\{c_{i}, \lambda\right\}=E$.

The constrained equal-losses rule, $C E L$, selects, for each $(N, c, E) \in \mathcal{D}$, the vector $\left(\max \left\{0, c_{i}-\right.\right.$ $\lambda\})_{i \in N}$, where $\lambda>0$ is chosen so that $\sum_{i \in N} \max \left\{0, c_{i}-\lambda\right\}=E$.

The Talmud rule is a hybrid of the above two. Precisely, for each $(N, c, E) \in \mathcal{D}$, it selects

$$
T(N, c, E)= \begin{cases}C E A\left(N, \frac{1}{2} c, E\right) & \text { if } E \leq \frac{1}{2} C \\ \frac{1}{2} c+C E L\left(N, \frac{1}{2} c, E-\frac{1}{2} C\right) & \text { if } E \geq \frac{1}{2} C\end{cases}
$$

Finally, the proportional rule, $P$, yields awards proportionally to claims, i.e., for each $(N, c, E) \in \mathcal{D}, P(N, c, E)=\frac{E}{C} \cdot c$.

In a (broadcasting sports) problem $(N, A) \in \mathcal{P}$, as formalized above, the issue is to allocate the aggregate audience in the tournament $(\|A\|)$ among the participating teams $(N)$. If we

\footnotetext{
${ }^{13}$ The reader is referred to Thomson $(2003,2015,2017)$ for excellent surveys of the sizable literature dealing with this model.
} 


\section{Daw}

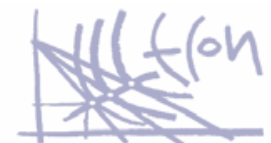

assume that each team claims the entire audience of the games it played $\left(\alpha_{i}(N, A)\right)$, then we obviously have a problem of adjudicating conflicting claims. Formally, we associate with each (broadcasting sports) problem $(N, A) \in \mathcal{P}$ a claims problem $\left(N, c^{A}, E^{A}\right) \in \mathcal{D}$ where $c_{i}^{A}=\alpha_{i}(N, A)$, for each $i \in N$, and $E^{A}=\|A\|$.

In Example 1 we have $E=4920$ and

\begin{tabular}{|c|c|c|c|}
\hline$i$ & 1 & 2 & 3 \\
\hline$c_{i}^{A}$ & 4460 & 2860 & 2520 \\
\hline$P_{i}\left(N, c^{A}, E^{A}\right)$ & 2230 & 1430 & 1260 \\
\hline$C E A_{i}\left(N, c^{A}, E^{A}\right)$ & 1640 & 1640 & 1640 \\
\hline$C E L_{i}\left(N, c^{A}, E^{A}\right)$ & 2820 & 1220 & 880 \\
\hline$T_{i}\left(N, c^{A}, E^{A}\right)$ & 2230 & 1430 & 1260 \\
\hline
\end{tabular}

The next result summarizes our findings in this section. The Talmud rule coincides with the proportional rule and the equal-split rule. The $C E A$ rule and the $C E L$ rule do not guarantee allocations within the core. It also states the stability properties of the above rules. It turns out that, of our four rules, only the proportional rule (or the Talmud rule, as they both coincide in this setting) guarantees allocations within the core. This is because, as mentioned above, the proportional rule yields the same outcomes as the equal-split rule.

Proposition 2 The following statements hold:

(a) For each $(N, A) \in \mathcal{P}, P\left(N, c^{A}, E^{A}\right)=T\left(N, c^{A}, E^{A}\right)=E S(N, A) \in C\left(N, v_{A}\right)$.

(b) There exists $(N, A) \in \mathcal{P}$ such that $C E A\left(N, c^{A}, E^{A}\right) \notin C\left(N, v_{A}\right)$.

(c) There exists $(N, A) \in \mathcal{P}$ such that $C E L\left(N, c^{A}, E^{A}\right) \notin C\left(N, v_{A}\right)$.

Proof. (a) For each $(N, A) \in \mathcal{P}, E^{A}=\frac{\sum_{i \in N} c_{i}^{A}}{2}$. Thus,

$$
P\left(N, c^{A}, E^{A}\right)=T\left(N, c^{A}, E^{A}\right)=\frac{c}{2}=E S(N, A) \in C\left(N, v_{A}\right) .
$$

(b) Let $(N, A) \in \mathcal{P}$ be such that $N=\{1,2,3,4\}$ and

$$
A=\left(\begin{array}{llll}
0 & 3 & 0 & 0 \\
3 & 0 & 0 & 0 \\
0 & 0 & 0 & 1 \\
0 & 0 & 1 & 0
\end{array}\right)
$$




\section{Daw}

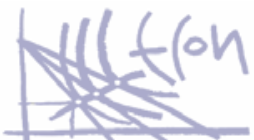

Then, $E^{A}=8, c^{A}=(6,6,2,2)$. Thus, $C E A\left(N, c^{A}, E^{A}\right)=(2,2,2,2)$. As

$$
C E A_{1}\left(N, c^{A}, E^{A}\right)+C E A_{2}\left(N, c^{A}, E^{A}\right)=4<6=a_{12}+a_{21},
$$

it follows from Proposition $1(b)$ that $C E A\left(N, c^{A}, E^{A}\right) \notin C\left(N, v_{A}\right)$.

(c) Let $(N, A) \in \mathcal{P}$ be such that $N=\{1,2,3,4\}$ and

$$
A=\left(\begin{array}{llll}
0 & 9 & 0 & 0 \\
9 & 0 & 0 & 0 \\
0 & 0 & 0 & 1 \\
0 & 0 & 1 & 0
\end{array}\right)
$$

Then, $E^{A}=20, c^{A}=(18,18,2,2)$ and $C E L\left(N, c^{A}, E^{A}\right)=(10,10,0,0)$. As

$$
C E L_{3}\left(N, c^{A}, E^{A}\right)+C E L_{4}\left(N, c^{A}, E^{A}\right)=0<2=a_{34}+a_{43},
$$

it follows from Proposition $1(b)$ that $C E L\left(N, c^{A}, E^{A}\right) \notin C\left(N, v_{A}\right)$

\section{The maximalist scenario for the fan effect and concede- and-divide}

In this section we consider the opposite scenario to that analyzed in the previous section. More precisely, we consider the maximalist scenario for the fan effect and assume that when somebody decides to watch a game, it is because he/she is a fan of one of the teams or because he/she is a fan of the specific sport being considered. In other words, nobody belongs to group 4, i.e., nobody is a joint fan of both teams in a game. In this scenario, we believe each team should receive the revenues generated by its fans, whereas the revenue coming from the generic sport fans should be divided equally among all teams. We may argue that this maximalist scenario is extreme and also that the minimalist scenario analyzed in the previous section was extreme in the opposite way. It will be nevertheless interesting to understand the two polar cases as they will represent meaningful lower and upper bounds for the amounts teams should get.

Formally, for each pair $i, j \in N$, with $i \neq j$, let

$$
a_{i j}=b_{0}+b_{i}+b_{j}+\varepsilon_{i j}
$$


where $b_{0}$ denotes the number of generic sport fans, $b_{k}$ denotes the number of fans of team $k=i, j$, and $\varepsilon_{i j}$ denotes the number of joint fans for the pair $\{i, j\}$. In this scenario, our aim is to minimize $\left\{\varepsilon_{i j}\right\}_{i, j \in N}$ (given the audience data).

Thus, we aim to solve

$$
\min _{b \in \mathbb{R}^{n+1}} \sum_{i, j \in N, i \neq j} \varepsilon_{i j}^{2}=\min _{b \in \mathbb{R}^{n+1}} \sum_{i, j \in N, i \neq j}\left(a_{i j}-b_{0}-b_{i}-b_{j}\right)^{2} .
$$

Unfortunately, the above problem cannot be solved. ${ }^{14}$ We then remove one of the teams $k \in N$, and consider the following minimization problem:

$$
\min _{b \in \mathbb{R}^{n}} \sum_{i, j \in N, i \neq j} \varepsilon_{i j}^{2}
$$

where

$$
\varepsilon_{i j}=\left\{\begin{array}{cc}
a_{i j}-b_{0}-b_{i}-b_{j} & \text { if } k \notin\{i, j\} \\
a_{i j}-b_{0}-b_{i} & \text { if } k=j \\
a_{i j}-b_{0}-b_{j} & \text { if } k=i
\end{array}\right.
$$

Let $\hat{b}_{0}$ and $\left\{\hat{b}_{i}\right\}_{i \in N \backslash\{k\}}$ denote the solutions to (2). Besides, for each pair $i, j \in N$, with $i \neq j$, we denote

$$
\hat{\varepsilon}_{i j}=\left\{\begin{array}{cc}
a_{i j}-\hat{b}_{0}-\hat{b}_{i}-\hat{b}_{j} & \text { if } k \notin\{i, j\} \\
a_{i j}-\hat{b}_{0}-\hat{b}_{i} & \text { if } k=j \\
a_{i j}-\hat{b}_{0}-\hat{b}_{j} & \text { if } k=i
\end{array}\right.
$$

We now impose the following principles to allocate $a_{i j}$ :

$(P 1) \hat{b}_{0}$ is divided equally among all teams. ${ }^{15}$

$(P 2) \hat{b}_{l}$ is assigned to team $l$, for each $l \in N \backslash\{k\}$.

$(P 3) \hat{\varepsilon}_{i j}$ is divided equally between teams $i$ and $j$, for each pair $i, j \in N$, with $i \neq j$.

\footnotetext{
${ }^{14}$ This is due to the fact that the minimization problem (1) coincides with the minimization problem induced by the $O L S$ estimator associated with the following regression model:

$$
Y=b_{0}+\sum_{i \in N} b_{i} X_{i}+\varepsilon
$$

where $Y$ is the audience of a game, $X_{i}$ is the team dummy variable (i.e., $X_{i}=1$ if team $i$ plays the game and 0 otherwise) and $\varepsilon$ is the error term. It is straightforward to see that, for each $k=1, \ldots, n, X_{k}=2_{A}-\sum_{i \in N \backslash\{k\}} X_{i}$, where $2_{A}$ is the vector with all coordinates equal to 2 . Thus, the problem involves colinearity.

${ }^{15}$ If, instead, we assume that it is divided equally between teams $i$ and $j$, nothing will change.
} 


\section{Dewe}

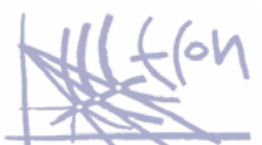

Applying those principles we can define a rule $R^{b, k}$ where, for each problem $(N, A) \in \mathcal{P}$ and each $i \in N$, the allocation for team $i$ is ${ }^{16}$

$$
R_{i}^{b, k}(N, A)=\left\{\begin{array}{cl}
(n-1) \widehat{b_{0}}+2(n-1) \widehat{b_{i}}+\sum_{j \in N \backslash\{i\}} \frac{\widehat{\varepsilon_{i j}}+\widehat{\varepsilon_{j i}}}{2} & \text { if } i \neq k \\
(n-1) \widehat{b_{0}}+\sum_{j \in N \backslash\{i\}} \frac{\widehat{\varepsilon_{i j}}+\widehat{\varepsilon_{j i}}}{2} & \text { if } i=k
\end{array}\right.
$$

One might argue that the above allocation would depend on $k$ (the removed team). The next theorem shows that this is not the case. It actually states that the allocation rule, so constructed, coincides with concede-and-divide.

Theorem 2 For each $(N, A) \in \mathcal{P}$ and each pair $i, k \in N$, let $R_{i}^{b, k}(N, A)$ be the allocation obtained by applying formula (3). Then,

$$
R_{i}^{b, k}(N, A)=\frac{(n-1) \alpha_{i}-\|A\|}{n-2}=C D_{i}(N, A) .
$$

Proof. We note first that the solution to the minimization problem (2) coincides with the $O L S$ estimator of the linear regression model where the set of dependent variables is $\left\{X_{i}\right\}_{i \in N \backslash\{k\}}$, and, thus, no colinearity occurs.

Given the linear regression model $V=b_{0}+\sum_{i \in S} b_{i} U_{i}+\varepsilon$, it is well known that the $O L S$ estimator is computed as

$$
\begin{aligned}
\left(\widehat{b_{i}}\right)_{i \in S} & =\operatorname{Cov}(U, U)^{-1} \operatorname{Cov}(U, V) \text { and } \\
\widehat{b_{0}} & =\bar{V}-\sum_{i \in S} \widehat{b}_{i} \overline{U_{i}}
\end{aligned}
$$

where

$$
\begin{aligned}
& \operatorname{Cov}(U, U)=\left(\operatorname{Cov}\left(U_{i}, U_{j}\right)\right)_{i, j \in S} \text { and } \\
& \operatorname{Cov}(U, V)=\left(\operatorname{Cov}\left(U_{i}, V\right)\right)_{i \in S}
\end{aligned}
$$

Besides, given two variables $U, V$ taking the values $\left\{\left(u_{k}, v_{k}\right)\right\}_{k=1}^{m}$ we have that

$$
\operatorname{Cov}(U, V)=\frac{\sum_{k=1}^{m} u_{k} v_{k}}{m}-\left(\frac{\sum_{k=1}^{m} u_{k}}{m}\right)\left(\frac{\sum_{k=1}^{m} v_{k}}{m}\right) .
$$

We now apply the previous expressions to our case.

\footnotetext{
${ }^{16}$ Note that each team plays $2(n-1)$ games.
} 
1. Let $i, j \in N$ with $i \neq j$.

$$
\begin{aligned}
\operatorname{Cov}\left(X_{i}, X_{j}\right) & =\frac{2}{n(n-1)}-\left(\frac{2(n-1)}{n(n-1)}\right)\left(\frac{2(n-1)}{n(n-1)}\right) \\
& =\frac{2}{n(n-1)}-\frac{4}{n^{2}}=\frac{2(2-n)}{n^{2}(n-1)} .
\end{aligned}
$$

2. Let $i \in N$.

$$
\begin{aligned}
\operatorname{Cov}\left(X_{i}, X_{i}\right) & =\frac{2(n-1)}{n(n-1)}-\left(\frac{2(n-1)}{n(n-1)}\right)\left(\frac{2(n-1)}{n(n-1)}\right) \\
& =\frac{2}{n}-\frac{4}{n^{2}}=\frac{2(n-2)}{n^{2}} .
\end{aligned}
$$

3. Let $i \in N$.

$$
\begin{aligned}
\operatorname{Cov}\left(X_{i}, Y\right) & =\frac{\alpha_{i}}{n(n-1)}-\left(\frac{2(n-1)}{n(n-1)}\right)\left(\frac{\|A\|}{n(n-1)}\right) \\
& =\frac{\alpha_{i}}{n(n-1)}-\frac{2\|A\|}{n^{2}(n-1)} \\
& =\frac{n \alpha_{i}-2\|A\|}{n^{2}(n-1)}=\left(\alpha_{i}-\frac{2\|A\|}{n}\right) \frac{1}{n(n-1)} .
\end{aligned}
$$

Then $\operatorname{Cov}(U, U)=\operatorname{Cov}\left(X_{i}, X_{j}\right)_{i, j \in N \backslash\{k\}}$ is a matrix of $(n-1) \times(n-1)$ dimension. It is not difficult to show that

$$
\operatorname{Cov}(U, U)^{-1}=\frac{n(n-1)}{2(n-2)}\left(\begin{array}{cccc}
2 & 1 & \ldots & 1 \\
1 & 2 & \ldots & 1 \\
1 & \ldots & \ldots & 1 \\
1 & 1 & 1 & 2
\end{array}\right)
$$

Besides,

$$
\operatorname{Cov}(U, V)=\frac{1}{n^{2}(n-1)}\left(\begin{array}{c}
n \alpha_{1}-2\|A\| \\
\ldots \\
n \alpha_{n}-2\|A\|
\end{array}\right)
$$

Because of (4), we have that, for each $j \in N \backslash\{k\}$,

$$
\begin{aligned}
\widehat{b_{j}} & =\frac{n(n-1)}{2(n-2)} \frac{1}{n^{2}(n-1)}\left[2\left(n \alpha_{j}-2\|A\|\right)+\sum_{i \in N \backslash\{j, k\}}\left(n \alpha_{i}-2\|A\|\right)\right] \\
& =\frac{1}{2(n-2) n}\left[2 n \alpha_{j}-4\|A\|+n \sum_{i \in N \backslash\{j, k\}} \alpha_{i}-2(n-2)\|A\|\right] \\
& =\frac{1}{2(n-2) n}\left[2 n \alpha_{j}+n \sum_{i \in N \backslash\{j, k\}} \alpha_{i}-2 n\|A\|\right] .
\end{aligned}
$$


As $\sum_{i \in N} \alpha_{i}=2\|A\|$, we have that

$$
\begin{aligned}
\widehat{b_{j}} & =\frac{1}{2(n-2) n}\left[2 n \alpha_{j}+n\left(2\|A\|-\left(\alpha_{j}+\alpha_{k}\right)\right)-2 n\|A\|\right] \\
& =\frac{1}{2(n-2) n}\left[2 n \alpha_{j}+2 n\|A\|-n\left(\alpha_{j}+\alpha_{k}\right)-2 n\|A\|\right] \\
& =\frac{1}{2(n-2) n}\left[n\left(\alpha_{j}-\alpha_{k}\right)\right]=\frac{\alpha_{j}-\alpha_{k}}{2(n-2)} .
\end{aligned}
$$

Furthermore,

$$
\begin{aligned}
\widehat{b_{0}} & =\bar{Y}-\sum_{j \in N \backslash\{k\}} \widehat{b_{j}} \overline{X_{j}}=\frac{\|A\|}{n(n-1)}-\sum_{j \in N \backslash\{k\}} \frac{\alpha_{j}-\alpha_{k}}{2(n-2)} \frac{2(n-1)}{n(n-1)} \\
& =\frac{\|A\|}{n(n-1)}-\sum_{j \in N \backslash\{k\}} \frac{\alpha_{j}-\alpha_{k}}{n(n-2)} \\
& =\frac{\|A\|}{n(n-1)}-\frac{1}{n(n-2)}\left[\sum_{j \in N \backslash\{k\}} \alpha_{j}-(n-1) \alpha_{k}\right] \\
& =\frac{\|A\|}{n(n-1)}-\frac{1}{n(n-2)}\left[2\|A\|-\alpha_{k}-(n-1) \alpha_{k}\right] \\
& =\frac{\|A\|}{n(n-1)}-\frac{2\|A\|}{n(n-2)}+\frac{\alpha_{k}}{n-2}=-\frac{\|A\|}{(n-1)(n-2)}+\frac{\alpha_{k}}{n-2} .
\end{aligned}
$$

Once we have estimated the parameters we have that

$$
\begin{array}{rr}
a_{i j}=\widehat{b_{0}}+\widehat{b_{i}}+\widehat{b_{j}}+\widehat{\varepsilon_{i j}} \quad \text { if } i, j \in N \backslash\{k\} \\
a_{i k}=\widehat{b_{0}}+\widehat{b_{i}}+\widehat{\varepsilon_{i k}} \quad \text { if } i \in N \backslash\{k\} \\
a_{k i}=\widehat{b_{0}}+\widehat{b_{i}}+\widehat{\varepsilon_{k i}} \quad \text { if } i \in N \backslash\{k\} .
\end{array}
$$

Given $i, j \in N \backslash\{k\}$,

$$
\begin{aligned}
\widehat{\varepsilon_{i j}} & =a_{i j}-\widehat{b_{0}}-\widehat{b_{i}}-\widehat{b_{j}}= \\
& =a_{i j}+\frac{\|A\|}{(n-1)(n-2)}-\frac{\alpha_{k}}{n-2}-\frac{\alpha_{i}-\alpha_{k}}{2(n-2)}-\frac{\alpha_{j}-\alpha_{k}}{2(n-2)} \\
& =a_{i j}+\frac{\|A\|}{(n-1)(n-2)}-\frac{\alpha_{i}+\alpha_{j}}{2(n-2)} .
\end{aligned}
$$

Given $i \in N \backslash\{k\}$,

$$
\begin{aligned}
\widehat{\varepsilon_{i k}} & =a_{i k}-\widehat{b_{0}}-\widehat{b_{i}}= \\
& =a_{i k}+\frac{\|A\|}{(n-1)(n-2)}-\frac{\alpha_{k}}{n-2}-\frac{\alpha_{i}-\alpha_{k}}{2(n-2)} \\
& =a_{i k}+\frac{\|A\|}{(n-1)(n-2)}-\frac{\alpha_{i}+\alpha_{k}}{2(n-2)} .
\end{aligned}
$$


Analogously, we have that

$$
\widehat{\varepsilon_{k i}}=a_{k i}+\frac{\|A\|}{(n-1)(n-2)}-\frac{\alpha_{i}+\alpha_{k}}{2(n-2)}
$$

Notice that, for each pair $i, j \in N$,

$$
\widehat{\varepsilon_{i j}}=a_{i j}+\frac{\|A\|}{(n-1)(n-2)}-\frac{\alpha_{i}+\alpha_{j}}{2(n-2)} .
$$

We now compute the rule $R_{i}^{b, k}(N, A)$ by applying principles $(P 1),(P 2)$ and $(P 3)$ in this regression. We consider two cases:

- Team $i \in N \backslash\{k\}$. The audience assigned to team $i$ is made of three components:

By $(P 1)$, team $i$ receives

$$
(n-1) \widehat{b_{0}}=-\frac{\|A\|}{n-2}+\frac{(n-1) \alpha_{k}}{n-2} .
$$

By $(P 2)$, team $i$ receives

$$
2(n-1) \widehat{b_{i}}=\frac{(n-1)\left(\alpha_{i}-\alpha_{k}\right)}{n-2} .
$$

By (P3), team $i$ receives

$$
\begin{aligned}
\sum_{j \in N \backslash\{i\}} \frac{\widehat{\varepsilon_{i j}}+\widehat{\varepsilon_{j i}}}{2} & =\frac{1}{2} \sum_{j \in N \backslash\{i\}}\left(a_{i j}+a_{j i}\right)+\frac{\|A\|}{(n-2)}-\frac{(n-1) \alpha_{i}+\sum_{j \in N \backslash\{i\}} \alpha_{j}}{2(n-2)} \\
& =\frac{\alpha_{i}}{2}+\frac{\|A\|}{n-2}-\frac{(n-1) \alpha_{i}+2\|A\|-\alpha_{i}}{2(n-2)} \\
& =\frac{\alpha_{i}}{2}+\frac{\|A\|}{(n-2)}-\frac{\alpha_{i}}{2}-\frac{\|A\|}{n-2}=0 .
\end{aligned}
$$

Thus, team $i$ receives

$$
\begin{aligned}
R_{i}^{b, k}(N, A) & =-\frac{\|A\|}{n-2}+\frac{(n-1) \alpha_{k}}{n-2}+\frac{(n-1)\left(\alpha_{i}-\alpha_{k}\right)}{n-2} \\
& =\frac{(n-1) \alpha_{i}-\|A\|}{n-2} .
\end{aligned}
$$

- Team $k$. The audience assigned to team $k$ is also made of three components:

By $(P 1)$, team $k$ receives

$$
(n-1) \widehat{b_{0}}=-\frac{\|A\|}{n-2}+\frac{(n-1) \alpha_{k}}{n-2} .
$$


By $(P 2)$, team $k$ receives nothing.

Analogously to the previous case, by $(P 3)$, team $k$ receives nothing.

Thus, team $k$ receives

$$
R_{k}^{P, k}(N, A)=\frac{(n-1) \alpha_{k}-\|A\|}{n-2} .
$$

Theorem 2 shows that the rule derived from (3), with the minimization problem (2), is precisely concede-and-divide, as introduced in Section 2.

\section{The axiomatic approach}

The previous two sections provided arguments to endorse, respectively, the two focal rules of this work. First, the equal-split rule was shown to coincide with the Shapley value (as well as the Nucleolus and the $\tau$-value) of a natural convex TU-game, thus guaranteeing stable outcomes (as formalized by the core of such a game). Second, concede-and-divide arose as the outcome of an optimization problem aiming to minimize the number of joint fans. In this section, we provide normative foundations for both rules.

The first axiom we consider says that if two teams have the same audiences, then they should receive the same amount.

Equal treatment of equals: For each $(N, A) \in \mathcal{P}$, and each pair $i, j \in N$ such that $a_{i k}=a_{j k}$, and $a_{k i}=a_{k j}$, for each $k \in N \backslash\{i, j\}$,

$$
R_{i}(N, A)=R_{j}(N, A)
$$

The second axiom says that revenues should be additive on $A$. Formally,

Additivity: For each pair $(N, A)$ and $\left(N, A^{\prime}\right) \in \mathcal{P}$

$$
R\left(N, A+A^{\prime}\right)=R(N, A)+R\left(N, A^{\prime}\right) .
$$

The third axiom says that if nobody watches a single game of a given team (i.e., the team has a null audience), then such a team gets no revenue. 


\section{Daw}

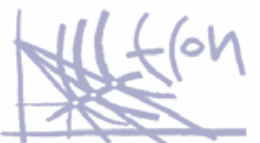

Null team: For each $(N, A) \in \mathcal{P}$, and each $i \in N$, such that $a_{i j}=0=a_{j i}$, for each $j \in N$,

$$
R_{i}(N, A)=0 .
$$

Alternatively, the next axiom says that if a team nullifies the audience of all the games it plays (for instance, due to some kind of boycott), then the allocation of such a team should decrease exactly by the total audience of such a team. ${ }^{17}$ Formally,

Nullifying team: For each $(N, A),\left(N, A^{\prime}\right) \in \mathcal{P}$ such that there exists $k \in N$ (the nullifying team) satisfying $a_{i j}^{\prime}=a_{i j}$ when $k \notin\{i, j\}$ and $a_{i j}^{\prime}=0$ when $k \in\{i, j\}$ we have that

$$
R_{k}\left(N, A^{\prime}\right)=R_{k}(N, A)-\alpha_{k}(A)
$$

The next result provides the characterizations of the two rules.

Theorem 3 The following statements hold:

(a) A rule satisfies equal treatment of equals, additivity and null team if and only if it is the equal-split rule.

(b) A rule satisfies equal treatment of equals and nullifying team if and only if it is concedeand-divide.

Proof. (a) It is not difficult to show that the equal-split rule satisfies the three axioms in the statement. Conversely, let $R$ be a rule satisfying them. Let $(N, A) \in \mathcal{P}$. For each pair $i, j \in N$, with $i \neq j$, let $A^{i j}$ denote the matrix with the following entries:

$$
a_{k l}^{i j}=\left\{\begin{array}{cc}
a_{i j} & \text { if }(k, l)=(i, j) \\
0 & \text { otherwise. }
\end{array}\right.
$$

Notice that $a_{j i}^{i j}=0$.

Let $k \in N$. By additivity,

$$
R_{k}(N, A)=\sum_{i, j \in N: i \neq j} R_{k}\left(N, A^{i j}\right)
$$

By null team, for each pair $i, j \in N$ with $i \neq j$, and for each $l \in N \backslash\{i, j\}$, we have $R_{l}\left(N, A^{i j}\right)=0$. Thus,

$$
R_{k}(N, A)=\sum_{l \in N \backslash\{k\}}\left[R_{k}\left(N, A^{l k}\right)+R_{k}\left(N, A^{k l}\right)\right]
$$

\footnotetext{
${ }^{17}$ A similar axiom was introduced in cooperative transferable utility games by van den Brink (2007).
} 


\section{Daw}

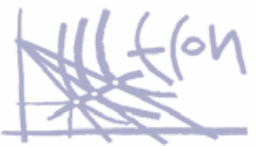

By equal treatment of equals, $R_{k}\left(N, A^{l k}\right)=R_{l}\left(N, A^{l k}\right)$. As $\left\|A^{l k}\right\|=a_{l k}$, we have that $R_{k}\left(N, A^{l k}\right)=\frac{a_{l k}}{2}$. Similarly, $R_{k}\left(N, A^{k l}\right)=\frac{a_{k l}}{2}$. Thus,

$$
R_{k}(N, A)=\sum_{l \in N \backslash\{k\}}\left[\frac{a_{l k}}{2}+\frac{a_{k l}}{2}\right]=\frac{\alpha_{k}}{2}=S_{k}(N, A) .
$$

(b) It is not difficult to show that concede-and-divide satisfies both axioms. Conversely, let $R$ be a rule satisfying the two axioms in the statement. Let $(N, A) \in \mathcal{P}$. Let $t(A)$ be the number of null teams in $(N, A)$. We proceed recursively on $t(A)$. Notice that $t(A) \in\{0,1, \ldots, n-2, n\}$.

Suppose first that $t(A)=n$. Then, $A=0_{N, N}$ (the matrix with all entries equal to 0 ). By equal treatment of equals, for each $i \in N$,

$$
R_{i}\left(N, 0_{N, N}\right)=0=C D_{i}\left(N, 0_{N, N}\right) .
$$

Suppose now that $t(A)=n-2$. Then, $A=A^{i j}+A^{j i}$ for some $i, j \in N$ and, therefore, $\alpha_{i}(N, A)=\alpha_{j}(N, A)=a_{i j}+a_{j i}$, whereas $\alpha_{k}(N, A)=0$ for each $k \in N \backslash\{i, j\}$. Then,

$$
C D_{k}(N, A)= \begin{cases}a_{i j}+a_{j i} & \text { if } k \in\{i, j\} \\ \frac{-\left(a_{i j}+a_{j i}\right)}{n-2} & \text { otherwise. }\end{cases}
$$

As $(N, A),\left(N, 0_{N, N}\right)$, and $k=i$ are under the hypothesis of nullifying team,

$$
0=R_{i}\left(N, 0_{N, N}\right)=R_{i}(N, A)-\left(a_{i j}+a_{j i}\right) .
$$

Thus, $R_{i}(N, A)=a_{i j}+a_{j i}$. Analogously, we can prove that $R_{j}(N, A)=a_{i j}+a_{j i}$.

By equal treatment of equals, we have that $R_{k}\left(N, A^{i j}\right)=R_{l}\left(N, A^{i j}\right)$, for each pair $k, l \in$ $N \backslash\{i, j\}$. Let $x$ denote such an amount. Then,

$$
a_{i j}+a_{j i}=\|A\|=\sum_{k \in N} R_{k}(N, A)=2\left(a_{i j}+a_{j i}\right)+(n-2) x
$$

from where it follows that $x=\frac{-\left(a_{i j}+a_{j i}\right)}{n-2}$.

Thus, $R(N, A)=C D(N, A)$, in this case too.

Assume now that $R$ coincides with $C D$ in problems with $r$ null players. We prove that both rules also coincide when we have $r-1$ null players.

Let $(N, A) \in \mathcal{P}$ be a problem with $r-1$ null players. Let $k$ be a no null player in $(N, A)$. Let $\left(N, A^{-k}\right)$ be the problem obtained from $A$ by nullifying team $k$. Namely $a_{i j}^{-k}=a_{i j}$ when 

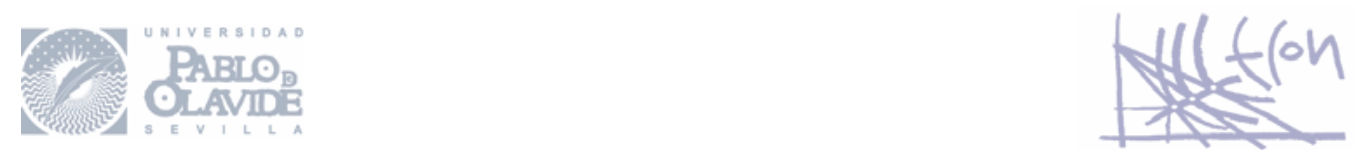

$k \notin\{i, j\}$ and $a_{i j}^{-k}=0$ when $k \in\{i, j\}$. As $A$ and $A^{-k}$ are under the hypothesis on the axiom of nullifying team, we deduce that

$$
R_{k}(N, A)=R_{k}\left(N, A^{-k}\right)+\alpha_{k}(N, A) \text { and } C D_{k}(N, A)=C D_{k}\left(N, A^{-k}\right)+\alpha_{k}(N, A) .
$$

As $k$ is a null player in $\left(N, A^{-k}\right)$, and $(N, A)$ has $r-1$ null players, $\left(N, A^{-k}\right)$ has $r$ null players. As $R$ and $C D$ coincide in problems with $r$ null players, we have that $R_{k}\left(N, A^{-k}\right)=$ $C D_{k}\left(N, A^{-k}\right)$. Thus, $R_{k}(N, A)=C D_{k}(N, A)$.

Let us denote by $D$ the set of null players in $(N, A)$. Then,

$$
\begin{aligned}
\sum_{i \in D} R_{i}(N, A) & =\|A\|-\sum_{i \in N \backslash D} R_{i}(N, A) \\
& =\|A\|-\sum_{i \in N \backslash D} C D_{i}(N, A) \\
& =\sum_{i \in D} C D_{i}(N, A) .
\end{aligned}
$$

As $R$ and $C D$ satisfy equal treatment of equals, all null teams in $(N, A)$ must receive the same according to both rules. Then, for each null player $i$ in $(N, A)$, we have that $R_{i}(N, A)=$ $C D_{i}(N, A)$.

Theorem 4 not only provides a characterization of our two focal rules, but also a common ground for them. More precisely, it states that both rules are characterized by the combination of equal treatment of equals, additivity, and a third axiom. ${ }^{18}$ This different axiom (null player in one case; nullifying player in the other case) formalizes the behavior of the rule with respect to somewhat peculiar teams (those with no viewers in one case; those making viewers vanish in the other case).

It turns out, nevertheless, that this only difference, reflected in the mentioned pair of axioms, is substantial as the axioms are incompatible. Namely, there is no rule satisfying both the null team axiom and the nullifying team axiom. Consider the problem $\left(N, A^{12}\right)$ defined as in the proof of Theorem 4, where $N=\{1,2,3\}$ and $a_{12}>0$. If $R$ satisfies null team we have that $R_{3}\left(N, A^{12}\right)=0$ and $R_{i}\left(N, 0_{N, N}\right)=0$ for each $i \in N$. Suppose that $R$ also satisfies nullifying team. Using arguments similar to the ones used in the proof of Theorem 4 we can deduce that $R_{1}\left(N, A^{12}\right)=R_{2}\left(N, A^{12}\right)=a_{12}$. Thus, $R_{3}\left(N, A^{12}\right)=-a_{12}$, which is a contradiction.

\footnotetext{
${ }^{18}$ Actually, additivity is not necessary in the characterization of concede-and-divide, although the rule does satisfy the axiom.
} 


\section{An empirical application}

In this section, we present an empirical application of our model resorting to La Liga, the Spanish Football League. ${ }^{19}$

La Liga is a standard round robin tournament involving 20 teams. Thus, each team plays 38 games, facing each time one of the other 19 teams (once home, another away). The 20 teams, and the overall audience (in millions) of each team during the last completed season (2016-2017), are listed in the first two columns of Table $1 .^{20}$ The last two columns contain the actual revenues they made (in millions of euros and in percentage terms). ${ }^{21}$

Insert Table 1 about here

As we can see, two teams dominated the sharing collecting a combined $22.9 \%$ of the pie.

Note that the total audience of the complete season is 178,5 millions, whereas the total revenue made was 1246, 9 millions of euros. Thus, in order to accommodate the premises of our model and identify total audience with total revenue, we have to assume that each viewer paid a pay-per-view fee of 7 euros (instead of only one) per game. This normalizing assumption, and the resulting scaling, will be implicit in the next tables describing the allocations.

Table 2 lists the allocations proposed by our two rules (equal-split and concede-and-divide), with the normalizing assumption mentioned above. They also appear in percentage levels.

\section{Insert Table 2 about here}

Several conclusions can be derived from Table 2. Maybe the most obvious one is that, contrary to what some might argue, the actual revenue sharing seems to be biased against the two powerhouses. More precisely, although the equal-split rule would recommend a somewhat

\footnotetext{
${ }^{19}$ http://www.laliga.es/en

${ }^{20}$ The source for most of the data provided here is Palco 23, the leading newspaper in economic information of the sport business in Spain. Palco 23 refers itself to Havas Sports and Entertainment as its source. See, for instance, https://www.palco23.com/marketing/la-lucha-por-la-audiencia-que-clubes-se-siguieron-mas-en-tv.html. These data are also confirmed by a report published in Marca, the leading Spanish newspaper in terms of daily readers, on the first fifth of the season. See, for instance, http://www.marca.com/futbol/laboratoriodatos/2017/05/22/5922fd72468aeb984e8b4612.html

${ }^{21}$ The source is La Liga's website. See, for instance, http://www.laliga.es/lfp/reparto-ingresos-audiovisuales
} 
Table 1. Audiences and revenues for the Spanish Football League in 2016/2017

\begin{tabular}{|l|r|r|r|}
\hline \multicolumn{1}{|c|}{ Teams } & \multicolumn{1}{c|}{$\begin{array}{c}\text { Total audience } \\
\text { (millions of viewers) }\end{array}$} & $\begin{array}{c}\text { Total allocation } \\
\text { (millions of euros) }\end{array}$ & $\begin{array}{c}\text { Relative allocation } \\
\text { (\%) }\end{array}$ \\
\hline Alavés & 10,71 & 39,3 & 3,2 \\
\hline Athletic Bilbao & 15,98 & 71,0 & 5,7 \\
\hline Atlético Madrid & 23,21 & 99,2 & 8,0 \\
\hline Barcelona & 40,04 & 146,2 & 11,7 \\
\hline Betis & 20,54 & 49,2 & 3,9 \\
\hline Celta & 13,54 & 51,4 & 4,1 \\
\hline Deportivo Coruña & 13,42 & 44,0 & 3,5 \\
\hline Eibar & 12,6 & 41,7 & 3,3 \\
\hline Español & 14,88 & 48,9 & 3,9 \\
\hline Granada & 13,08 & 44,6 & 3,6 \\
\hline Las Palmas & 16,86 & 44,0 & 3,5 \\
\hline Leganés & 11,18 & 39,3 & 3,2 \\
\hline Málaga & 16,82 & 55,6 & 4,5 \\
\hline Osasuna & 11,44 & 43,0 & 3,4 \\
\hline Real Madrid & 43,61 & 140,1 & 11,2 \\
\hline Real Sociedad & 18,14 & 53,5 & 4,3 \\
\hline Sevilla & 19,26 & 65,9 & 5,3 \\
\hline Sporting & 11,18 & 41,7 & 3,3 \\
\hline Valencia & 15,02 & 67,4 & 5,4 \\
\hline Villareal & 15,47 & & 3,9 \\
\hline
\end{tabular}


Table 2. The equal-split and concede-and-divide outcomes for the Spanish Footbalk League in 2016/2017

\begin{tabular}{|l|r|r|r|r|}
\hline TEAM & ES & CD & EN \\
\hline Alavés & 37,41 & 3,0 & 9,70 & 0,8 \\
\hline Athletic Bilbao & 55,81 & 4,5 & 48,56 & 3,9 \\
\hline Atlético Madrid & 81,07 & 6,5 & 101,87 & 8,2 \\
\hline Barcelona & 139,85 & 11,2 & 225,97 & 18,1 \\
\hline Betis & 71,74 & 5,8 & 82,18 & 6,6 \\
\hline Celta & 47,29 & 3,8 & 30,57 & 2,5 \\
\hline Deportivo Coruña & 46,87 & 3,8 & 29,68 & 2,4 \\
\hline Eibar & 44,01 & 3,5 & 23,64 & 1,9 \\
\hline Español & 51,97 & 4,2 & 40,45 & 3,2 \\
\hline Granada & 45,68 & 3,7 & 27,18 & 2,2 \\
\hline Las Palmas & 58,89 & 4,7 & 55,05 & 4,4 \\
\hline Leganés & 39,05 & 3,1 & 13,17 & 1,1 \\
\hline Málaga & 58,75 & 4,7 & 54,75 & 4,4 \\
\hline Osasuna & 39,96 & 3,2 & 15,08 & 1,2 \\
\hline Real Madrid & 152,32 & 12,2 & 252,29 & 20,2 \\
\hline Real Sociedad & 63,36 & 5,1 & 64,49 & 5,2 \\
\hline Sevilla & 67,27 & 5,4 & 72,75 & 5,8 \\
\hline Sporting & 39,05 & 3,1 & 43,17 & 1,1 \\
\hline Valencia & 52,46 & 4,2 & 44,80 & 3,3 \\
\hline Villareal & 54,03 & 4,3 & 3,6 \\
\hline
\end{tabular}



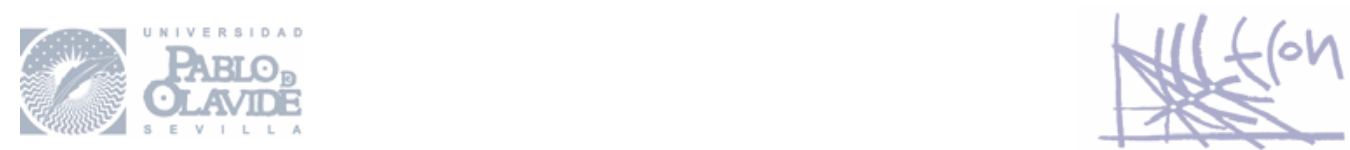

similar aggregated allocation for them (close to one fourth of the pie), concede-and-divide would recommend for them almost two fifths of the pie. More precisely, Real Madrid would be obtaining $12.2 \%$ with the equal-split rule and $20.2 \%$ with concede-and-divide. Barcelona would also go up considerably with concede-and-divide (from $11.7 \%$ to $18.1 \%$ ), although it would go down with the equal-split rule (from $11.7 \%$ to $11.2 \%$ ).

Another conclusion is that eight teams (Alavés, Athletic, Celta, Leganés, Osasuna, Sporting, Valencia and Villareal) are favored by the actual allocation, in the sense that the amount they get is above the amounts suggested by the two rules. Seven teams (Atlético de Madrid, Barcelona, Deportivo, Eibar, Español, Granada and Malaga) obtain amounts between those suggested by the two rules. Finally, beyond Real Madrid, there are four teams (Betis, Las Palmas, Real Sociedad and Sevilla) that obtain amounts below those suggested by the two rules. The case of Betis is remarkable, as the allocation yields $3.9 \%$, whereas the two rules would recommend $5.8 \%$ and $6.6 \%$, respectively.

It has been argued that an extremely unequal sharing of the broadcasting revenues would be detrimental for the overall quality of the tournament. Some even go further claiming that a system with unequal shares of revenue, widening the gap between clubs, might violate EU competition law. ${ }^{22}$ Clubs with higher earnings will be able to attract more playing talent. Eventually, this will make them prevail (overwhelmingly) in their national tournaments, winning easily most of the games. Likewise, teams with lower earnings will become less competitive, eventually giving up while playing against the richest teams (preserving their key players for the ensuing more balanced games against other peer teams). This will render most of the games in the tournament uninteresting (even for the fan base of the rich teams). Because of this, one might argue that a sharing process based on performance might not be that different from a sharing process based on audiences. This statement can be rejected with the data presented above. For instance, Betis appears fourth in audience figures, whereas it ended fifteenth in the league standings; Las Palmas appears seventh in audience figures, whereas it ended fourteenth in the league standings; Villareal appears tenth in audience figures, whereas it ended fifth in

\footnotetext{
${ }^{22}$ In late 2014, the so-called FASFE (an organization consisting of groups of fans, club members, and minority shareholders of several Spanish professional football clubs) and the International Soccer Centre (a movement that aims to obtain more balanced and transparent football and basketball competitions in Spain) filed an antitrust complaint with the European Commission against the Spanish National Professional Football League.
} 

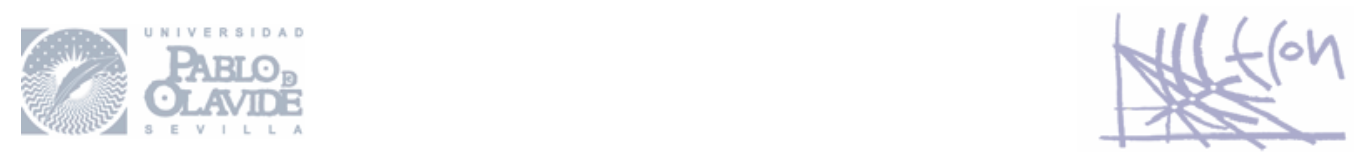

the league standings; Alavés appears last in audience figures, whereas it ended ninth in the league standings;

\begin{tabular}{|c|c|c|c|}
\hline $\begin{array}{c}\text { Criteria } \rightarrow \\
\text { Country }\end{array}$ & Egalitarian & $\begin{array}{c}\text { League performance: } \\
\text { Scoring, .. }\end{array}$ & $\begin{array}{c}\text { Social performance: } \\
\text { TV audience, ... }\end{array}$ \\
\hline England & $50 \%$ & $25 \%$ & $25 \%$ \\
\hline Germany & & $100 \%$ & $30 \%$ \\
\hline Italy & $40 \%$ & $30 \%$ & $25 \%$ \\
\hline Spain (new) & $50 \%$ & $25 \%$ & \\
\hline
\end{tabular}

Table 3. Hybrid revenue sharing in the most important European football leagues.

To account for the above (at least, partially), we consider alternative schemes with our database described above. More precisely, we present in what follows different mixed schemes in which a portion of the overall revenue is equally divided, another is proportionally divided according to scoring performance, and the residual is divided according to one of our two rules (thus, only taking into account the audiences). Note that this is indeed what happens in the most important European football leagues, as described in Table 3. La Liga itself implemented a new scheme along those lines for the past season (2016-2017) in which, as shown by the table, half of the overall revenue will be shared equally, whereas one quarter will be shared according to league performance and the remaining quarter according to social performance. ${ }^{23}$

Table 4 summarizes the outcomes that our rules would yield when modified to endorse the hybrid scheme implemented by La Liga. More precisely, we assume that half of the overall revenue will be shared equally (that would represent 31.2 million euros for each team), whereas one quarter will be shared according to league performance and the remaining quarter according to social performance (where we apply our two rules). By league performance, La Liga refers to the places at the end of the previous five seasons (where a zero score is given to those teams that played in the second division, or below, in one of those years). One quarter of the budget is then allocated proportionally to those 5-year standings. By social performance, La Liga assigns one third (of the corresponding one quarter) proportionally to the revenues generated from ticket

\footnotetext{
${ }^{23}$ The full details of this new scheme, which was actually sanctioned by the Spanish government, appeared in the Official Bulletin of the Spanish State on May 1st, 2015. The numbers provided in Table 1 are supposed to reflect this scheme.
} 

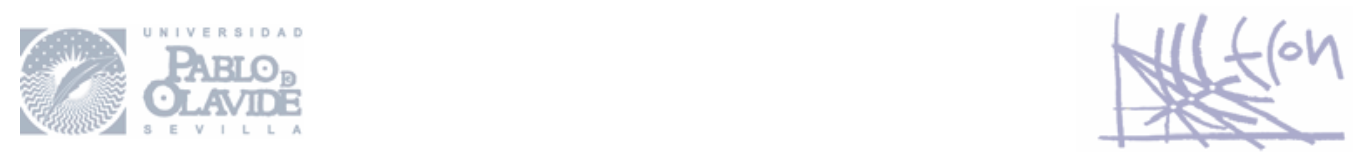

sales in the last five seasons. ${ }^{24}$ The other two thirds (of that one quarter) are supposed to be assigned according to audiences. We will then consider our equal-split and concede-and-divide rules for that portion of the budget. More precisely, the second and third columns of Table 4 are the result of aggregating (for each team) the fixed amount (31.2 million), the proportional amounts to league performance and ticket sales, and the amount suggested by each rule for the division of the remaining one sixth of the budget.

\section{Insert Table 4 about here}

An obvious aspect that one can observe from Table 4 is that the hybrid schemes become more egalitarian. More precisely, under the full-fledged equal-split rule, the two powerhouses were obtaining (combined) $23.4 \%$ of the pie. The hybrid scheme lowers this to $20.8 \%$. Under the full-fledged concede-and-divide, the two powerhouses were obtaining (combined) 38.3\%, which now drastically moves down (under the hybrid scheme) to $23.3 \%$.

Table 4 now shows that eight teams (Barcelona, Celta, Granada, Leganés, Málaga, Osasuna, Sporting and Valencia) are favored by the actual allocation, in the sense that the amount they get is above the amounts suggested by the two hybrid schemes. Four teams (Alavés, Deportivo, Eibar and Real Madrid) obtain amounts between those suggested by the two rules. The remaining eight teams (Athletic, Atlético de Madrid, Betis, Español, Las Palmas, Real Sociedad, Sevilla and Villareal) obtain amounts below those suggested by the two hybrid schemes.

A final aspect is that the two hybrid schemes produce much more similar allocations to the existing one. In the case of the equal-split (hybrid) scheme only one team (Barcelona) varies more than $1 \%$ with respect to the existing scheme, and the overall variance is $6.2 \%$. In the case of the concede-and-divide (hybrid) scheme, all teams vary less than $1 \%$ with respect to the existing scheme (Real Sociedad is the one varying most, with $0.9 \%$ ), and the overall variance is $6.7 \%$.

\footnotetext{
${ }^{24}$ For this, we consider data on season tickets for the last two seasons, which are the only ones available (again, obtained from Palco 23). See, for instance, https://www.palco23.com/clubes/los-clubes-arrancan-la-ligasantander-con-cerca-de-600-000-abonados.html and https://www.palco23.com/clubes/los-clubes-de-primera-ysegunda-rozan-los-800000-abonados-en-2017-2018.html
} 
Table 4. Revenues and hybrid equal-split and concede-and-divide outcomes for the Spanish Football League in 2016/2017

\begin{tabular}{|c|c|c|c|c|c|c|}
\hline TEAM & Allocation & (Hybrid) ES & (Hybrid) CD & Allocation (\%) & (Hybrid) ES (\%) & (Hybrid) CD (\%) \\
\hline Alavés & 39,3 & 41,4 & 36,7 & 3,2 & 3,3 & 2,9 \\
\hline Athletic Bilbao & 71,0 & 72,3 & 71,1 & 5,7 & 5,8 & 5,7 \\
\hline Atlético Madrid & $\quad 99, \overline{2}$ & 99,7 & 103,2 & & 8,0 & 8,3 \\
\hline Barcelona & 146,2 & 128,7 & 143,1 & 11,7 & $\infty$ & 11,5 \\
\hline Betis & 49,2 & 52,4 & 54,1 & 3,9 & 4,2 & 4,3 \\
\hline Celta & 51,4 & 51,3 & 48,5 & 4,1 & 4,1 & 3,9 \\
\hline Deportivo Coruña & 44,0 & 44,7 & 41,9 & 3,5 & 3,6 & 3,4 \\
\hline Eibar & 41,7 & 43,3 & 39,9 & 3,3 & 3,5 & 3,2 \\
\hline Español & 48,9 & 51,8 & 49,9 & 3,9 & 4,2 & 4,0 \\
\hline Granada & 44,6 & 43,5 & 40,4 & 3,6 & 3,5 & 3,2 \\
\hline Las Palmas & 44,0 & 46,5 & 45,9 & 3,5 & 3,7 & 3,7 \\
\hline Leganés & 39,3 & 38,9 & 34,6 & 3,2 & 3,1 & 2,8 \\
\hline Málaga & 55,6 & 54,8 & 54,1 & 4,5 & 4,4 & 4,3 \\
\hline Osasuna & 43,0 & 40,8 & 36,7 & 3,4 & 3,3 & 2,9 \\
\hline Real Madrid & 140,1 & 130,3 & 146,9 & 11,2 & 10,4 & 11,8 \\
\hline Real Sociedad & 53,5 & 64,4 & 64,5 & 4,3 & 5,2 & 5,2 \\
\hline Sevilla & 65,9 & 73,2 & 74,1 & 5,3 & 5,9 & 5,9 \\
\hline Sporting & 41,7 & 41,1 & 36,8 & 3,3 & 3,3 & 2,9 \\
\hline Valencia & 67,4 & 61,9 & 60,1 & 5,4 & 5,0 & 4,8 \\
\hline Villareal & 60,9 & 65,7 & 64,2 & 4,9 & 5,3 & 5,1 \\
\hline
\end{tabular}




\section{Discussion}

We have presented a stylized model to deal with the problem of sharing the revenues from broadcasting sport events. We have provided normative, empirical and game-theoretical foundations for rules sharing each game's revenues equally or proportionally among the participating teams. Both rules have distinguishing merits. One (concede-and-divide) is supported by an intuitive procedure aiming to reflect the (potentially different) fan base of each team. Another (the equal-split rule) is supported by a powerful (and normatively appealing) stability property preventing secessions from participating players.

We have also provided as a case study an empirical application deriving what both rules would suggest for the Spanish Football League (La Liga). Hybrid schemes in which our rules are only used to share one sixth of the budget, whereas the remaining budget is allocated according to the criteria of La Liga, yield close outcomes to the current allocation being implemented by the Spanish National Professional Football League.

It is left for further research to enrich the model in plausible ways. For instance, some games are offered for free (in non-subscription channels), instead of pay per view. That might influence the audience numbers. In our case study (La Liga), not all teams are broadcasted under that option. And the broadcasting rights for those games are negotiated independently. Thus, it might well make sense to talk about two different budgets: one coming from subscription channels (to which all teams have access) and another coming from non-subscription channels (to which not all teams have access, and which might be associated to different audience figures).

Similarly, several games might be broadcasted simultaneously, which might reduce the number of viewers for some games. And if all games are broadcasted in exclusive time windows (as it happens, for instance, in our case study), prime time is only awarded to some games. All these aspects, which have been ignored in our model, might have an important impact on audience figures.

Finally, we could also consider other forms of tournaments. For instance, an elimination (as opposed to a round robin) tournament divided into successive rounds in which, as rounds progress, the number of competitors decreases. This is a popular form in international competitions (e.g., Football World Cups) and its analysis would require a different model, able to include concepts from graph theory. ${ }^{25}$

\footnotetext{
${ }^{25}$ For instance, the teams competing in the round of 16 at the UEFA Champions League, will increase their
} 


\section{References}

[1] Adams, W.J., Yellen, J.L., 1973, Commodity Bundling and the Burden of Monopoly. Quarterly Journal of Economics 90, 475-98.

[2] Atkinson, S.E., Stanley, L.R., Tschirhart, J., 1988. Revenue sharing as an incentive in an agency problem: An example from the National Football League. RAND Journal of Economics 19, 27-43.

[3] Aumann R.J., Maschler M., 1985. Game theoretic analysis of a bankruptcy problem from the Talmud. Journal of Economic Theory 36, 195-213.

[4] Bergantiños, G., Moreno-Ternero, J.D., 2015. The axiomatic approach to the problem of sharing the revenue from museum passes. Games and Economic Behavior 89, 78-92.

[5] Brink van den, R., 2007. Null or nullifying players: The difference between the Shapley value and equal division solutions. Journal of Economic Theory 136, 767-775

[6] El Hodiri, M., Quirk, J., 1971. An economic model of a professional sports league. Journal of Political Economy 79, 1302-1319.

[7] Flores-Szwagrzak, K., Treibich, R., 2017. Teamwork and Individual Productivity. Mimeo. Copenhagen Business School.

[8] Ginsburgh, V., Zang, I., 2003. The museum pass game and its value. Games and Economic Behavior 43, 322-325.

[9] Hansen, B.O., Tvede, M., 2016. Revenue Sharing in European Football Leagues: A Theoretical Analysis. Chapter 12 in A.A. Pinto et al., (eds.), Trends in Mathematical Economics. Springer.

[10] Késenne, S., 2000. Revenue sharing and competitive balance in professional team sports. Journal of Sports Economics 1, 56-65.

[11] Littlechild, S., Owen. G., 1973, A simple expression for the Shapley value in a special case, Management Science 20, 370-372.

base payment by receiving an additional 6 million euros fee, with the quarter-finalists then picking up 6.5 million apiece and the semi-finalists pocketing 7.5 million each. 

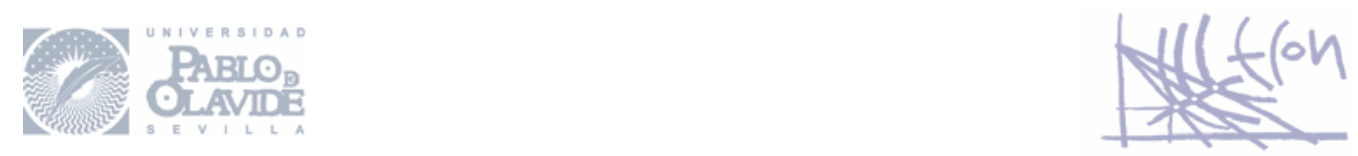

[12] van den Nouweland, A., Borm, P., van Golstein Brouwers, W., Groot Bruinderink, R., Tijs, S., 1996. A Game Theoretic Approach to Problems in Telecommunication. Management Science 42, 294-303.

[13] Moreno-Ternero, J., Roemer, J., 2006. Impartiality, priority and solidarity in the theory of justice. Econometrica 74, 1419-1427.

[14] Moreno-Ternero, J., Roemer, J., 2012. A common ground for resource and welfare egalitarianism. Games and Economic Behavior 75, 832-841.

[15] Moreno-Ternero, J., Thomson, W., 2017. Between(s) Aristotle and the Talmud. Mimeo. University of Rochester.

[16] O’Neill, B., 1982. A problem of rights arbitration from the Talmud. Mathematical Social Sciences 2, 345-371.

[17] Rottenberg, S., 1956. The baseball players' labor market. Journal of Political Economy 64, $242-258$.

[18] Shapley, L., 1953. A value for n-person games, in Contributions to the Theory of Games II (Annals of Mathematics Studies 28), ed. by H.W. Kuhn and A.W. Tucker, Princeton: Princeton University Press, 307-317.

[19] Thomson, W., 2003. Axiomatic and game-theoretic analysis of bankruptcy and taxation problems: a survey, Mathematical Social Sciences 45, 249-297.

[20] Thomson W., 2014. Fair allocation. Princeton University Press. Forthcoming.

[21] Thomson W., 2015. Axiomatic and game-theoretic analysis of bankruptcy and taxation problems: an update. Mathematical Social Sciences 74, 41-59.

[22] Thomson W., 2017. How to divide when there isn't enough: from Aristotle, the Talmud, and Maimonides to the axiomatics of resource allocation, Econometric Society Monograph. Forthcoming. 


\section{Appendix that is not part of the submission for publication}

To save space, we have included in this appendix, which is not for publication a more detailed axiomatic analysis of the two rules considered in this paper.

\section{Appendix}

We first provide a list of axioms and study which ones are satisfied by each rule. Then, we characterize both rules using some of them.

The first axiom we consider says that if two teams have the same audiences, then they should receive the same amount.

Equal treatment of equals: For each $(N, A) \in \mathcal{P}$, and each pair $i, j \in N$ such that $a_{i k}=a_{j k}$, and $a_{k i}=a_{k j}$, for each $k \in N \backslash\{i, j\}$,

$$
R_{i}(N, A)=R_{j}(N, A)
$$

The second axiom says that revenues should be additive on $A$. Formally,

Additivity: For each pair $(N, A)$ and $\left(N, A^{\prime}\right) \in \mathcal{P}$

$$
R\left(N, A+A^{\prime}\right)=R(N, A)+R\left(N, A^{\prime}\right) .
$$

The third axiom says that if nobody watches a single game of a given team (i.e., the team has a null audience), then such a team gets no revenue.

Null team: For each $(N, A) \in \mathcal{P}$, and each $i \in N$, such that $a_{i j}=0=a_{j i}$, for each $j \in N$,

$$
R_{i}(N, A)=0
$$

Alternatively, the next axiom says that if a team nullifies the audience of all the games it plays (for instance, due to some kind of boycott), then the allocation of such a team should decrease exactly by the total audience of such a team. ${ }^{26}$ Formally,

\footnotetext{
${ }^{26} \mathrm{~A}$ similar axiom was introduced in cooperative transferable utility games by van den Brink (2007).
} 

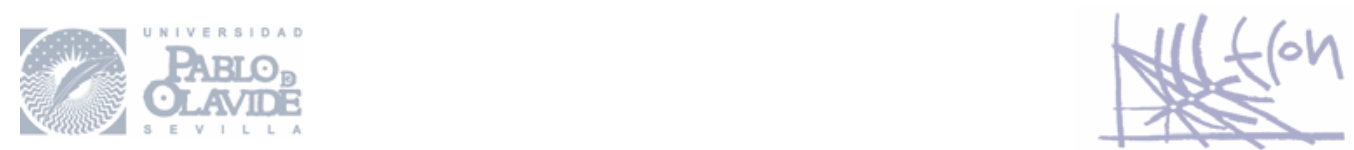

Nullifying team: For each $(N, A),\left(N, A^{\prime}\right) \in \mathcal{P}$ such that there exists $k \in N$ (the nullifying team) satisfying $a_{i j}^{\prime}=a_{i j}$ when $k \notin\{i, j\}$ and $a_{i j}^{\prime}=0$ when $k \in\{i, j\}$ we have that

$$
R_{k}\left(N, A^{\prime}\right)=R_{k}(N, A)-\alpha_{k}(A)
$$

The next axiom says that the allocation should be in the core of the game $v_{A}$, described in Section 3.

Core selection: For each $(N, A) \in \mathcal{P}$,

$$
R(N, A) \in C\left(N, v_{A}\right) .
$$

The next axiom says that no team should receive negative awards.

Non negativity. For each $(N, A) \in \mathcal{P}$ and $i \in N$,

$$
R_{i}(N, A) \geq 0
$$

The next axiom says that if the audience of team $i$ is, game by game, not smaller than the audience of team $j$, then team $i$ could not receive less than team $j$.

Monotonicity: For each $(N, A) \in \mathcal{P}$ and each pair $i, j \in N$, such that, for each $k \in$ $N \backslash\{i, j\}, a_{i k} \geq a_{j k}$ and $a_{k i} \geq a_{k j}$ we have that

$$
R_{i}(N, A) \geq R_{j}(N, A)
$$

The next axiom says that each team should receive, at most, the total audience of the games played by the team.

Maximum aspirations: For each $(N, A) \in \mathcal{P}$ and each $i \in N$,

$$
R_{i}(N, A) \leq \alpha_{i}(N, A)
$$

The next axiom refers to the incremental effect of adding a single additional viewer to a game. It states that the additional revenue should be shared equally among the involved teams in such a game. Formally,

Equal sharing of additional viewers: For each pair $(N, A),(N, \hat{A}) \in \mathcal{P}$ such that $a_{i j}=$ $\hat{a}_{i j}$, for each pair $(i, j) \neq\left(i_{0}, j_{0}\right)$, and $a_{i_{0}, j_{0}}+1=\hat{a}_{i_{0}, j_{0}}$,

$$
R_{i_{0}}(N, \hat{A})-R_{i_{0}}(N, A)=R_{j_{0}}(N, \hat{A})-R_{j_{0}}(N, A)
$$

We now study which axioms are satisfied by each rule. 

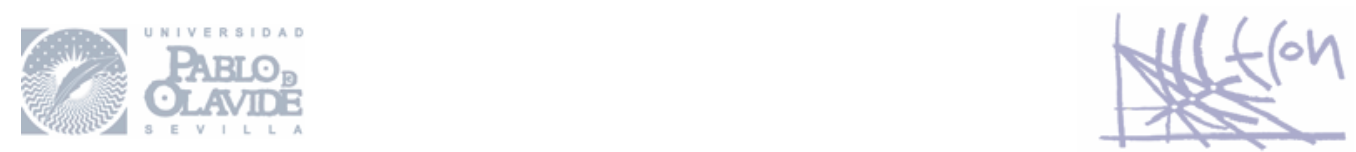

Proposition 3 The equal-split rule satisfies equal treatment of equals, additivity, null team, monotonicity, core selection, non negativity, maximum aspirations, and equal sharing of additional viewers, but violates nullifying team.

Proof. It is trivial to show that $S$ satisfies equal treatment of equals, null team, monotonicity, non negativity, and maximum aspirations. We have already seen that $S$ satisfies core selection. Additivity is a consequence of the fact that, for each pair $(N, A),\left(N, A^{\prime}\right) \in \mathcal{P}$, and each $i \in N$, $\alpha_{i}\left(N, A+A^{\prime}\right)=\alpha_{i}(N, A)+\alpha_{i}\left(N, A^{\prime}\right)$. Similarly, equal sharing of additional viewers is a consequence of the fact that $\alpha_{i}(N, \hat{A})=\alpha_{i}(N, A)+1$ when $i \in\left\{i_{0}, j_{0}\right\}$.

As for nullifying team, let $(N, A)$ be such that $N=\{1,2,3\}$ and $a_{i j}=10$ for each pair $i, j \in N, i \neq j$. Let $\left(N, A^{\prime}\right)$ be obtained from $A$ by nullifying team 3 . Namely, $a_{12}^{\prime}=a_{21}^{\prime}=10$ and $a_{i j}^{\prime}=0$ otherwise. Then $\alpha(N, A)=(40,40,40)$ and $\alpha\left(N, A^{\prime}\right)=(20,20,0)$. Hence, $E S(N, A)=(20,20,20)$ and $E S\left(N, A^{\prime}\right)=(10,10,0)$, which shows that $S$ does not satisfy nullifying team, as $S_{3}\left(N, A^{\prime}\right)=0 \neq-20=S_{3}(N, A)-\alpha_{3}(A)$.

Proposition 4 Concede-and-divide satisfies equal treatment of equals, additivity, nullifying team, monotonicity, maximum aspirations, and equal sharing of additional viewers, but violates null team, core selection and non negativity.

Proof. It is trivial to show that $C D$ satisfies equal treatment of equals, monotonicity and maximum aspirations. Additivity is a consequence of the fact that, for each pair $(N, A),\left(N, A^{\prime}\right) \in \mathcal{P}$, and each $i \in N,\left\|A+A^{\prime}\right\|=\|A\|+\left\|A^{\prime}\right\|$ and $\alpha_{i}\left(N, A+A^{\prime}\right)=\alpha_{i}(N, A)+\alpha_{i}\left(N, A^{\prime}\right)$.

Let $(N, A),\left(N, A^{\prime}\right) \in \mathcal{P}$ and $k \in N$ be as in the definition of nullifying team. Then,

$$
\begin{aligned}
C D_{k}\left(N, A^{\prime}\right) & =\alpha_{k}\left(N, A^{\prime}\right)-\frac{\sum_{j, k \in N \backslash\{i\}}\left(a_{j k}^{\prime}+a_{k j}^{\prime}\right)}{n-2} \\
& =-\frac{\sum_{j, k \in N \backslash\{i\}}\left(a_{j k}+a_{k j}\right)}{n-2} \\
& =\alpha_{k}(N, A)-\frac{\sum_{j, k \in N \backslash\{i\}}\left(a_{j k}+a_{k j}\right)}{n-2}-\alpha_{k}(N, A) \\
& =C D_{k}(N, A)-\alpha_{k}(N, A) .
\end{aligned}
$$

Then, $C D$ satisfies nullifying team. 


\section{Dew}

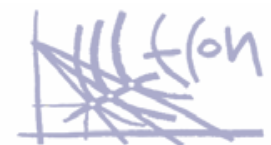

Let $(N, A),(N, \hat{A}) \in \mathcal{P}$ and $\left(i_{0}, j_{0}\right)$ as in the definition of equal sharing of additional viewers. As $\|\hat{A}\|=\|A\|+1$ and $\alpha_{i}(N, \hat{A})=\alpha_{i}(N, A)+1$ when $i \in\left\{i_{0}, j_{0}\right\}$, we have that, for each $i \in\left\{i_{0}, j_{0}\right\}$,

$$
\begin{aligned}
R_{i}(N, \hat{A})-R_{i}(N, A) & =\frac{(n-1) \alpha_{i}(N, \hat{A})-\|\hat{A}\|}{n-2}-\frac{(n-1) \alpha_{i}(N, A)-\|A\|}{n-2} \\
& =\frac{(n-1)\left[\alpha_{i}(N, A)+1\right]-[\|A\|+1]}{n-2}-\frac{(n-1) \alpha_{i}(N, A)-\|A\|}{n-2} \\
& =1 .
\end{aligned}
$$

Then, $C D$ satisfies equal sharing of additional viewers.

As for the remaining axioms, let $(N, A) \in \mathcal{P}$ be such that $N=\{1,2,3\}, a_{12}=a_{21}=10$ and $a_{i j}=0$ otherwise. Then, $\|A\|=20$ and $\alpha=(20,20,0)$. Hence $C D(N, A)=(20,20,-20)$. From here, it follows that $C D$ does not satisfy null team, because $a_{3 i}=a_{i 3}=0$, for each $i \in N$, but $C D_{3}(N, a)=-20 \neq 0$. Similarly, $C D$ does not satisfy non negativity because $C D_{3}(N, a)=-20<0$, and core selection because $C D_{3}(N, A)=-20<0=v_{A}(3)$.

We have seen that $C D$ could provide negative awards. This fact is not counterintuitive at all in the maximalist scenario for the fan effect. Consider, for instance, a league with three teams where team 1 has 600 followers, team 2 has also 600, and team 3 has not followers. Besides, no follower of team 1 and 2 wants to watch the games of team 3. This situation induces a problem where $a_{12}=a_{21}=1200$ and $a_{i j}=0$ otherwise. Under this assumption, team 1 should receive 2400 because it plays four games in the league and with a contribution of 600 fans to each game. The same happens with team 2. Nevertheless, the contribution of team 3 to the league is negative because it has no fans and, moreover, the other teams' fans do not want to watch the games of team 3 .

Theorem 4 The following statements hold:

(a) A rule satisfies equal treatment of equals, additivity and null team if and only if it is the equal-split rule.

(b) A rule satisfies equal sharing of additional viewers, additivity and null team if and only if it is the equal-split rule.

(c) A rule satisfies equal treatment of equals and nullifying team if and only if it is concedeand-divide. 


\section{Dew}

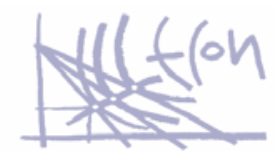

Proof. (a) By Proposition 3, the equal-split rule satisfies the three axioms in the statement. Conversely, let $R$ be a rule satisfying them. Let $(N, A) \in \mathcal{P}$. For each pair $i, j \in N$, with $i \neq j$, let $A^{i j}$ denote the matrix with the following entries:

$$
a_{k l}^{i j}=\left\{\begin{array}{cc}
a_{i j} & \text { if }(k, l)=(i, j) \\
0 & \text { otherwise }
\end{array}\right.
$$

Notice that $a_{j i}^{i j}=0$.

Let $k \in N$. By additivity,

$$
R_{k}(N, A)=\sum_{i, j \in N: i \neq j} R_{k}\left(N, A^{i j}\right)
$$

By null team, for each pair $i, j \in N$ with $i \neq j$, and for each $l \in N \backslash\{i, j\}$, we have $R_{l}\left(N, A^{i j}\right)=0$. Thus,

$$
R_{k}(N, A)=\sum_{l \in N \backslash\{k\}}\left[R_{k}\left(N, A^{l k}\right)+R_{k}\left(N, A^{k l}\right)\right]
$$

By equal treatment of equals, $R_{k}\left(N, A^{l k}\right)=R_{l}\left(N, A^{l k}\right)$. As $\left\|A^{l k}\right\|=a_{l k}$, we have that $R_{k}\left(N, A^{l k}\right)=\frac{a_{l k}}{2}$. Similarly, $R_{k}\left(N, A^{k l}\right)=\frac{a_{k l}}{2}$. Thus,

$$
R_{k}(N, A)=\sum_{l \in N \backslash\{k\}}\left[\frac{a_{l k}}{2}+\frac{a_{k l}}{2}\right]=\frac{\alpha_{k}}{2}=S_{k}(N, A) .
$$

(b) By Proposition 3, the equal-split rule satisfies the three axioms in the statement. Conversely, let $R$ be a rule satisfying them. Let $(N, A) \in \mathcal{P}$ and $k \in N$. As in the proof of $(a)$, it follows, by additivity and null team, that

$$
R_{k}(N, A)=\sum_{l \in N \backslash\{k\}}\left[R_{k}\left(N, A^{l k}\right)+R_{k}\left(N, A^{k l}\right)\right] .
$$

Let $0_{N, N}$ be the matrix with the same dimension of $A$ and all entries equal to 0 . By null team, $R_{j}\left(N, 0_{N, N}\right)=0$, for each $j \in N$. By equal sharing of additional viewers,

$$
\begin{aligned}
R_{k}\left(N, A^{l k}\right) & =R_{k}\left(N, A^{l k}\right)-R_{k}\left(N, 0_{N, N}\right) \\
& =R_{l}\left(N, A^{l k}\right)-R_{l}\left(N, 0_{N, N}\right) \\
& =R_{l}\left(N, A^{l k}\right) .
\end{aligned}
$$

From here, an analogous argument to that in the proof of $(a)$ allows to deduce too that $R(N, A)=\operatorname{ES}(N, A)$. 


\section{Dewe}

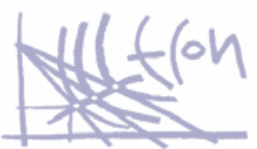

(c) By Proposition 4, concede-and-divide satisfies both axioms. Conversely, let $R$ be a rule satisfying the two axioms in the statement. Let $(N, A) \in \mathcal{P}$. Let $t(A)$ be the number of null teams in $(N, A)$. We proceed recursively on $t(A)$. Notice that $t(A) \in\{0,1, \ldots, n-2, n\}$.

Suppose first that $t(A)=n$. Then, $A=0_{N, N}$ (the matrix with all entries equal to 0 ). By equal treatment of equals, for each $i \in N$,

$$
R_{i}\left(N, 0_{N, N}\right)=0=C D_{i}\left(N, 0_{N, N}\right) .
$$

Suppose now that $t(A)=n-2$. Then, $A=A^{i j}+A^{j i}$ for some $i, j \in N$ and, therefore, $\alpha_{i}(N, A)=\alpha_{j}(N, A)=a_{i j}+a_{j i}$, whereas $\alpha_{k}(N, A)=0$ for each $k \in N \backslash\{i, j\}$. Then,

$$
C D_{k}(N, A)= \begin{cases}a_{i j}+a_{j i} & \text { if } k \in\{i, j\} \\ \frac{-\left(a_{i j}+a_{j i}\right)}{n-2} & \text { otherwise. }\end{cases}
$$

As $(N, A),\left(N, 0_{N, N}\right)$, and $k=i$ are under the hypothesis of nullifying team,

$$
0=R_{i}\left(N, 0_{N, N}\right)=R_{i}(N, A)-\left(a_{i j}+a_{j i}\right)
$$

Thus, $R_{i}(N, A)=a_{i j}+a_{j i}$. Analogously, we can prove that $R_{j}(N, A)=a_{i j}+a_{j i}$.

By equal treatment of equals, we have that $R_{k}\left(N, A^{i j}\right)=R_{l}\left(N, A^{i j}\right)$, for each pair $k, l \in$ $N \backslash\{i, j\}$. Let $x$ denote such an amount. Then,

$$
a_{i j}+a_{j i}=\|A\|=\sum_{k \in N} R_{k}(N, A)=2\left(a_{i j}+a_{j i}\right)+(n-2) x,
$$

from where it follows that $x=\frac{-\left(a_{i j}+a_{j i}\right)}{n-2}$.

Thus, $R(N, A)=C D(N, A)$, in this case too.

Assume now that $R$ coincides with $C D$ in problems with $r$ null players. We prove that both rules also coincide when we have $r-1$ null players.

Let $(N, A) \in \mathcal{P}$ be a problem with $r-1$ null players. Let $k$ be a no null player in $(N, A)$. Let $\left(N, A^{-k}\right)$ be the problem obtained from $A$ by nullifying team $k$. Namely $a_{i j}^{-k}=a_{i j}$ when $k \notin\{i, j\}$ and $a_{i j}^{-k}=0$ when $k \in\{i, j\}$. As $A$ and $A^{-k}$ are under the hypothesis on the axiom of nullifying team, we deduce that

$$
R_{k}(N, A)=R_{k}\left(N, A^{-k}\right)+\alpha_{k}(N, A) \text { and } C D_{k}(N, A)=C D_{k}\left(N, A^{-k}\right)+\alpha_{k}(N, A) .
$$

As $k$ is a null player in $\left(N, A^{-k}\right)$, and $(N, A)$ has $r-1$ null players, $\left(N, A^{-k}\right)$ has $r$ null players. As $R$ and $C D$ coincide in problems with $r$ null players, we have that $R_{k}\left(N, A^{-k}\right)=$ $C D_{k}\left(N, A^{-k}\right)$. Thus, $R_{k}(N, A)=C D_{k}(N, A)$. 


\section{Dewe:}

Let us denote by $D$ the set of null players in $(N, A)$. Then,

$$
\begin{aligned}
\sum_{i \in D} R_{i}(N, A) & =\|A\|-\sum_{i \in N \backslash D} R_{i}(N, A) \\
& =\|A\|-\sum_{i \in N \backslash D} C D_{i}(N, A) \\
& =\sum_{i \in D} C D_{i}(N, A) .
\end{aligned}
$$

As $R$ and $C D$ satisfy equal treatment of equals, all null teams in $(N, A)$ must receive the same according to both rules. Then, for each null player $i$ in $(N, A)$, we have that $R_{i}(N, A)=$ $C D_{i}(N, A)$.

Remark 1 The axioms of Theorem 4 are independent.

Let $R^{1}$ be the rule in which, for each game $(i, j) \in N \times N$, the revenue goes to the team with the lowest number of the two. Namely, for each problem $(N, A) \in \mathcal{P}$, and each $i \in N$,

$$
R_{i}^{1}(N, A)=\sum_{j \in N: j>i}\left(a_{i j}+a_{j i}\right) .
$$

$R^{1}$ satisfies null team and additivity, but not equal treatment of equals and equal sharing of additional viewers.

The rule that divides the total audience equally among the teams satisfies equal treatment of equals, equal sharing of additional viewers and additivity, but not null team.

Let $R^{2}$ be the rule that, for each pair $i, j \in N$, divides the audience $a_{i j}$ between teams $i$ and $j$ proportionally to their audiences in the games played agains the other teams. ${ }^{27}$ Namely, for each problem $(N, A) \in \mathcal{P}$, and $i \in N$,

$$
R_{i}^{2}(N, A)=\sum_{j \in N \backslash\{i\}} \frac{\sum_{k \in N \backslash\{i, j\}}\left(a_{i k}+a_{k i}\right)}{\sum_{k \in N \backslash\{i, j\}}\left(a_{i k}+a_{k i}\right)+\sum_{k \in N \backslash\{i, j\}}\left(a_{j k}+a_{k j}\right)}\left[a_{i j}+a_{j i}\right] .
$$

$R^{2}$ satisfies equal treatment of equals, equal sharing of additional viewers, and null team, but not additivity.

The equal-split rule satisfies equal treatment of equals but fails nullifying team.

Finally, we define the rule $R^{3}$ such that, for each problem $\left(N, A^{i j}\right) \in \mathcal{P}$, and $k \in N$,

$$
R_{k}^{3}\left(N, A^{i j}\right)= \begin{cases}a_{i j} & \text { if } k \in\{i, j\} \\ -a_{i j} & \text { if } k=\min \{l: l \in N \backslash\{i, j\}\} \\ 0 & \text { otherwise }\end{cases}
$$

\footnotetext{
${ }^{27}$ If such other audiences are both 0 , we divide equally.
} 
We extend $R^{3}$ to all problems using additivity. Namely, $R^{3}(N, A)=\sum_{i, j \in N: i \neq j} R^{3}\left(N, A^{i j}\right)$. $R^{3}$ satisfies nullifying team but fails equal treatment of equals.

Table 5 below summarizes the performance of both rules with respect to the axioms introduced in this section. The combination of the axioms with an asterisk in their cells characterizes the rule. The same happens for the plus symbol.

\begin{tabular}{|l|c|c|}
\hline Properties & equal-split & Concede-and-Divide \\
\hline Equal treatment of equals & YES* & YES* \\
\hline Additivity & YES$^{*+}$ & YES \\
\hline Null team & YES $^{*+}$ & NO \\
\hline Nullifying team & NO & YES* \\
\hline Core selection & YES & NO \\
\hline Non negativity & YES & NO \\
\hline Monotonicity & YES & YES \\
\hline Maximum aspirations & YES & YES \\
\hline Equal sharing of additional viewers & YES & YES \\
\hline
\end{tabular}

Table 5: Axiomatic Analysis. 